\title{
SENSITIVITY OF MESOSCALE GRAVITY WAVES TO THE BAROCLINICITY OF JET-FRONT SYSTEMS
}

\author{
A Thesis \\ by \\ SHUGUANG WANG \\ Submitted to the Office of Graduate Studies of \\ Texas A\&M University \\ in partial fulfillment of the requirements for the degree of \\ MASTER OF SCIENCE
}

December 2005

Major Subject: Atmospheric Sciences 


\title{
SENSITIVITY OF MESOSCALE GRAVITY WAVES TO THE BAROCLINICITY OF JET-FRONT SYSTEMS
}

\author{
A Thesis \\ by \\ SHUGUANG WANG \\ Submitted to the Office of Graduate Studies of \\ Texas A\&M University \\ in partial fulfillment of the requirements for the degree of \\ MASTER OF SCIENCE
}

Approved by:

Chair of Committee,

Fuqing Zhang

Committee Members,

Craig C. Epifanio

John W. Nielsen-Gammon

Ping Chang

Head of Department, $\quad$ Richard E. Orville

December 2005

Major Subject: Atmospheric Sciences 


\author{
ABSTRACT \\ Sensitivity of Mesoscale Gravity Waves to the Baroclinicity \\ of Jet-front Systems. (December 2005) \\ Shuguang Wang, B.S., Nanjing University \\ Chair of Advisory Committee: Dr. Fuqing Zhang
}

To investigate the generation of mesoscale gravity waves from upper-tropospheric jet-front systems, five different life cycles of baroclinic waves are simulated with a highresolution mesoscale model (MM5 with 10-km grid spacing). The baroclinicity (and the growth rates) of the baroclinic waves differs significantly among these five experiments as a result of using different tropospheric static stability and tropopause geometry for the initial two-dimensional baroclinic jet. After a short initial adjustment, the baroclinic waves in each experiment grow nearly linearly for as long as five days before the final nonlinear growth stage.

Vertically propagating mesoscale gravity waves are generated universally in the exit region of the upper-tropospheric jet streaks near the tropopause level at the later stage of quasi-linear growth and the early stage of nonlinear growth of baroclinic waves. The synoptic environments of gravity waves are consistent with previous studies of typical mesoscale gravity waves. The low-stratospheric gravity waves generated from the upper-level jet streak in these experiments have a horizontal wavelength of 75-175 km and an intrinsic frequency 3-10 times of the Coriolis parameter. The intrinsic frequency of these gravity waves appears to be directly correlated with the growth rate and the strength of the baroclinic waves. In general, the faster the growth rate of the baroclinic 
waves, the higher the intrinsic frequency of the gravity waves. Similar frequencies of the gravity waves are found in experiments with smaller quasi-linear growth rates of baroclinic waves but with significantly different initial tropospheric static stability and tropopause geometry.

The residuals of the nonlinear balance equation are used to assess the flow imbalance. It is also suggested that growth of imbalance is directly related to the growth rate of baroclinic waves and thus the frequency of primary gravity waves of interest. Diagnosis of flow imbalance suggests that balance adjustment, as a generalization of geostrophic adjustment, may be responsible for the generation of the gravity waves in the upper-tropospheric jet-front systems. 


\section{ACKNOWLEDGMENTS}

My foremost thanks go to my thesis adviser, Dr. Fuqing Zhang. This thesis would not have been possible without his continuous support. I thank him for his patience and encouragement that carried me through difficult times, and for his insights and suggestions that helped to shape my research skills.

I would also like to thank my other committee members, Dr. John NielsenGammon, Dr. Craig Epifanio and Dr. Ping Chang, for their expertise. Special thanks go to Dr. Nielsen-Gammon for his insightful discussions on experimental design and final results.

I also want to thank my labmates, Altug Aksoy, Daniel Hawblitzel, Ellie Meng, Naifang Bei, Yonghui Lin and Jason Sippel. I greatly appreciate Daniel Hawblitzel for his generous time in proofreading.

I thank my family for supporting me through all these years. Last but not least, I want to thank my girlfriend, Liang Liang, for all the support that she gave me while I have been working on this thesis. 


\section{TABLE OF CONTENTS}

Page

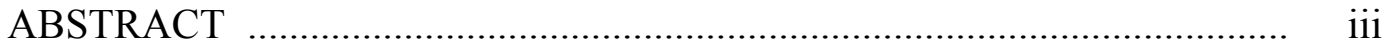

ACKNOWLEDGMENTS .................................................................. $\mathrm{v}$

TABLE OF CONTENTS ............................................................... vi

LIST OF TABLES .................................................................... viii

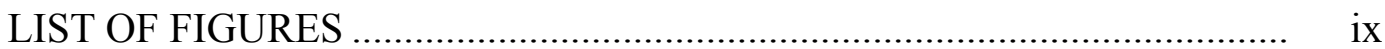

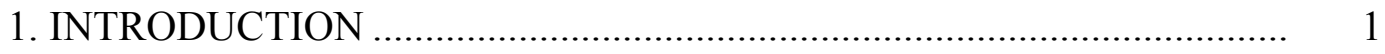

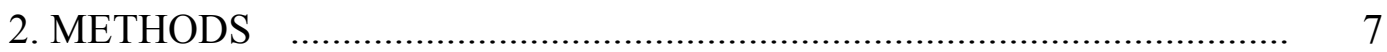

2.1 Initial Conditions ..................................................................... 7

2.2 Experimental Design................................................................... 11

3. CONTROL SIMULATION AND Z04 REVISITED ............................... 15

4. SENSITIVITY OF GRAVITY WAVES TO THE BACK GROUND BAROCLINICITY .......................................................... 28

4.1 Overview of simulated baroclinic waves........................................ 28

4.2 Overview of gravity waves in the five cases ................................. 38

4.3 Sensitivity of gravity wave characteristics to the baroclinic growth rates.............................................................. 47

4.3.1 Sensitivity to the slope of the initial tropopause.................. 47

4.3.2 Sensitivity to the tropospheric static stability ..................... 48

4.4 Gravity waves in the experiment with a lower tropospheric stability and a flatter initial tropopause........................................ 49

4.5 Summary $\quad$...................................................................... 50

5. FLOW IMBALANCE AND BALANCE ADJUSTMENT ...................... 52

5.1 Overview of flow imbalance diagnosis........................................ 52

5.2 Sensitivity of flow imbalance to baroclinic growth rate.................. 55

6. SUMMARY AND DISCUSSION................................................... 61 
Page

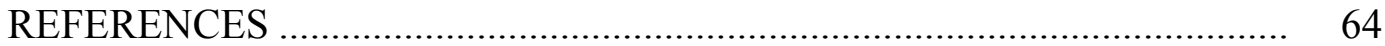

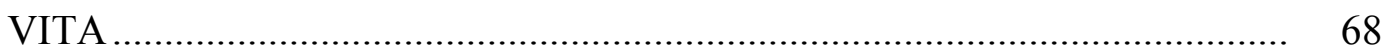




\section{LIST OF TABLES}

TABLE

Page

1 Summary of the five experiments. The first column is experiments.

The second and third columns are the tropopause geometry parameters. The fourth column is $P V_{T}$ used in the $2 \mathrm{D}$ inversion.

The fifth column is the maximum zonal wind speed. The sixth and seventh columns are start times of Domains 2 and 3. The last column is the total integration time.

2 Summary of characteristics for baroclinic waves for five experiments. Column 1 is the list of experiments. Column 2 lists the times of maximum imbalance when surface and $300 \mathrm{hPa}$ are shown in Figs. 10-11. Columns 3 and 4 are the maximum wind speeds at $300 \mathrm{hp}$ and minimum sea level pressure (MSLP) at these times. Column 5 is the time mature gravity waves are shown in Fig. 16. Column 6 is MSLP at the times listed in Column 5. Column 7 is the wavelength of the baroclinic wave. Columns 8 and 9 list the averaged quasi-linear growth rates and the period during which the averaged qiasi-linear growth rates are estimated.

3 Summary of wave characteristics for gravity waves for five experiments. Row 2 is the times when GWs are shown in Fig. 15. Rows 3-6 are horizontal wavelengths with the standard deviation, vertical wavelengths with the standard deviation, intrinsic frequency and phase speed $c_{i}$ derived from dispersion relation.

Rows 7-9 are mean wind speeds for GWs, ground relative phase speeds of GWs and mean flow relative phase speed based on columns 7 and 8. Row 10 is the growth rate of $\triangle \mathrm{NBE}$ calculated from Fig 22. 


\section{LIST OF FIGURES}

\section{FIGURE}

1 Initial conditions of CNTL from west to east in the middle of the Domain 1. PV anomalies (blue thin lines, ci $=0.1 \mathrm{PVU}$, negative value dashed), meridional winds $(\mathrm{ci}=2.5 \mathrm{~m} / \mathrm{s})$ green thin lines, negative value dashed), 1.5PVU potential vorticity (brown thick line) and potential temperatures (red thin lines, ci=6K). The distance between tick marks is $1000 \mathrm{~km}$.

2 Initial conditions for 5 experiments. Vertical cross section of initial potential temperature (thin line, $\mathrm{ci}=6 \mathrm{~K}$ ) and zonal velocity (thick line, $\mathrm{ci}=10 \mathrm{~m} \mathrm{~s}^{-1}$ ) for the initial basic-state jet. The gray thick line denotes the location of the tropopause where potential vorticity equals 1.5 PVU. )

3 Vertical velocities adapted from Fig. 6 in Z04. CNTL simulated potential temperature (thick line, $\mathrm{ci}=5 \mathrm{~K}$ ) and vertical velocity (solid, positive; dashed, negative; $\mathrm{ci}=2 \times 10^{-3} \mathrm{~m} / \mathrm{s}$; values $>5 \times 10^{-3} \mathrm{~m} / \mathrm{s}$ shaded) at the $13-\mathrm{km}$ level valid at (a) 102 and (b) $108 \mathrm{~h}$, and along the cross section AB valid at (c) 102 and (d) $108 \mathrm{~h}$. The straight line AB in (a) and (b) denotes the location of the cross section in (c) and (d). Contours of potential temperature greater than $340 \mathrm{~K}$ are generally flat but dense in the stratosphere and were thus not plotted in (c) and (d). The distance between tick marks in (a) and (b) is $100 \mathrm{~km}$. )

4 CNTL simulated 8-km pressure (thick line, $\mathrm{ci}=4 \mathrm{hPa}$ ), the residual of the nonlinear balance equation (shaded, solid, positive; dashed, negative; $c i=1 \times 10^{-9} \mathrm{~s}^{-2}$ ), and wind speed over $40 \mathrm{~m} / \mathrm{s}$ (bold contours, ci=5m/s) valid at (a) $78 \mathrm{~h}$, (b) $84 \mathrm{~h}$, (c) $90 \mathrm{~h}$, (d) $96 \mathrm{~h}$, (e) $102 \mathrm{~h}$, and (f) $108 \mathrm{~h}$ The straight line DE denotes the location of the cross section plotted in Fig. 11. The distance between tick marks is $300 \mathrm{~km}$. Adapted from Fig. 9 in Z04.

5 Potential temperature $\theta(\mathrm{ci}=5 \mathrm{~K}), Q$ vector and geopotential $\phi$ (ci $\left.=1000 \mathrm{~m}^{2} \mathrm{~s}^{2}\right)$ at $7 \mathrm{~km}$. Adapted from RSS94 Fig. 13.

6 CNTL simulated flow pattern at $6 \mathrm{~km}$ at $90 \mathrm{~h}(\mathrm{a}), 96 \mathrm{~h}(\mathrm{~b}), 102 \mathrm{~h}(\mathrm{c})$, $108 \mathrm{~h}(\mathrm{~d})$. Pressure (dashed lines, ci $=6 \mathrm{hPa}$ ), potential temperature $\theta$ (red solid lines, ci=5K), 1.5 PVU (brown thick lines) and wind speed (black lines, ci $=5 \mathrm{~m} / \mathrm{s}$ starts at $40 \mathrm{~m} / \mathrm{s}$ ). The distance between tick marks is $300 \mathrm{~km}$. 
FIGURE

7 CNTL simulated imbalanced flow at $6 \mathrm{~km}$ at $90 \mathrm{~h}(\mathrm{a}), 96 \mathrm{~h}(\mathrm{~b})$, $102 \mathrm{~h}(\mathrm{c})$ and $108 \mathrm{~h}(\mathrm{~d})$. Imbalance $\triangle N B E$ (shaded, solid, positive; dashed, negative; $c i=1 \times 10^{-9} \mathrm{~s}^{-2}$ ), $\theta$ (red solid lines, ci=5K), vertical velocity(blue thin lines, solid, positive; dashed, negative; $\mathrm{ci}=0.5 \mathrm{~cm} / \mathrm{s}$ in (a), $\mathrm{ci}=1.0 \mathrm{~cm} / \mathrm{s}$ in (b), ci=1.5 $\mathrm{cm} / \mathrm{s}$ in $(\mathrm{c}), \mathrm{ci}=2 \mathrm{~cm} / \mathrm{s}$ in (d)); and 1.5PVU (brown thick lines). The distance between tick marks is $300 \mathrm{~km}$.

8 Imbalance on tropopause denoted by 1.5PVU plane. $\triangle N B E$ (shaded, solid, positive; dashed, negative; ci= $1 \times 10^{-9} \mathrm{~s}^{-2}$ ) and geopotential (blue solid lines, $\mathrm{ci}=800 \mathrm{~m}$ ). The distance between tick marks is $300 \mathrm{~km}$.

9 Surface features for all the five cases. Potential temperature $\theta$ (red thin lines, $\mathrm{ci}=8 \mathrm{~K}$ ) and sea level pressure (Blue thin lines, $\mathrm{ci}=6 \mathrm{hPa}$ ). Green boxes denote the area in Figs. 15-18. The distance between tick marks is $300 \mathrm{~km}$.

10 Upper level jet. Isotachs (values greater than $35 / \mathrm{ms} \mathrm{light} \mathrm{shaded,}$ dark shaded regions are values greater than $55 \mathrm{~m} / \mathrm{s}$ for a-h, $65 / \mathrm{ms}$ for $\mathrm{i}, \mathrm{j}$ ), wind vector, geopotential (thick lines, $\mathrm{ci}=100 \mathrm{~m}$ ) and $\theta$ (thin lines, ci $=5 \mathrm{k}$ ) on $300 \mathrm{hPa}$. The distance between tick marks is $300 \mathrm{~km}$.

11 Divergence pattern 9-12 hours before gravity waves are plotted in Fig. 15. Divergence (thin line; solid and shaded, positive; dashed, negative; $\mathrm{ci}=2 \times 10^{-6} \mathrm{~s}^{-1}$ ), pressure (thick line, $\mathrm{ci}=2 \mathrm{hPa}$ ) and wind vectors. The distance between tick marks is $300 \mathrm{~km}$.

12 Divergence pattern at times gravity waves are displayed in Fig. 15. Divergence (thin line; solid and shaded, positive; dashed, negative; $\mathrm{ci}=2 \times 10^{-6} \mathrm{~s}^{-1}$ ), pressure (thick line, $\mathrm{ci}=2 \mathrm{hPa}$ ) and wind vectors. The distance between tick marks is $300 \mathrm{~km}$.

13 (a) Time series of MSLP (minimum sea level pressure) in Domain 2 for 5 experiments. (b) Minimum perturbation pressure for the cyclone in Domain $1(\mathrm{hPa})$. (c) Overall perturbation kinetic energy from surface to upper levels $(15 \mathrm{~km})$.

14 Growth rates in terms of (a) perturbation pressure and (b) perturbation kinetic energy for five experiments. Stars indicate the timing for each case in Fig. 20. Big circles indicate the timing in Fig. 15. 
15 Vertical velocities (solid, positive; dashed, negative; $\mathrm{ci}=2 \times 10^{-3} \mathrm{~m} / \mathrm{s}$; values $>5 \times 10^{-3} \mathrm{~m} / \mathrm{s}$ shaded) and $\theta$ (blue thick line, ci $=5 \mathrm{~K}$ ) on $13 \mathrm{~km}$ in Domain 3. Timing of plots is the same as in Fig. 10. The area is indicated by green box in Figs. 9-12. The distance between tick marks is $100 \mathrm{~km}$.

16 Vertical velocities (solid, positive; dashed, negative; $\mathrm{ci}=$ $5 \times 10^{-3} \mathrm{~m} / \mathrm{s}$; values $>5 \times 10^{-3} \mathrm{~m} / \mathrm{s}$ shaded) and $\theta$ (red thick line, $\mathrm{ci}=5 \mathrm{~K})$ along cross sections indicated in Fig. 15.

17 Perturbation vertical velocities $w^{\prime}$ (solid, positive; dashed, negative; $\mathrm{ci}=1 \times 10^{-3} \mathrm{~m} / \mathrm{s}$; values $>0.5 \times 10^{-3} \mathrm{~m} / \mathrm{s}$ shaded) and $\theta$ (blue thick line, $\mathrm{ci}=5 \mathrm{~K}$ ) on $13 \mathrm{~km}$. The distance between tick marks is $100 \mathrm{~km}$.

18 Perturbation vertical velocities $w^{\prime}$ (solid, positive; dashed, negative; $\mathrm{ci}=2 \times 10^{-3} \mathrm{~m} / \mathrm{s}$ ) and $\theta$ (thick lines, $\mathrm{ci}=5 \mathrm{~K}$ ) along cross sections indicated in Fig. 15.

19 Response function (15) for the filter used in Figures 17, 18 and 24.....

$20 \triangle N B E$ (shaded, solid, positive; dashed, negative. $\mathrm{ci}=0.01 \times 10^{-8} \mathrm{~s}^{-2}$ ), pressure (blue solid lines, $\mathrm{ci}=6 \mathrm{hPa}$ ) and $\theta$ (red lines, $\mathrm{ci}=5 \mathrm{~K}$ ) at $7 \mathrm{~km} \mathrm{9-12} \mathrm{hours} \mathrm{before} \mathrm{we} \mathrm{shown} \mathrm{GWs} \mathrm{in} \mathrm{Fig.} \mathrm{15.} \mathrm{Timings} \mathrm{are} \mathrm{also}$ summarized in Table 2 . The distance between tick marks is $300 \mathrm{~km} . .$.

$21 \Delta N B E$ (shaded, solid, positive; dashed, negative. $\mathrm{ci}=0.01 \times 10^{-8} \mathrm{~s}^{-2}$ ) and potential temperature (red lines, $\mathrm{ci}=5 \mathrm{~K}$ ) at the cross sections indicated by straight lines in Fig. 20.

22 Time series of averaged $\triangle N B E\left(10^{-8} \mathrm{~s}^{-2}\right)$ at 10 points with largest values for 5 cases.

$23 \triangle N B E$ (shaded, solid, positive; dashed, negative. $\mathrm{ci}=$ $0.01 \times 10^{-8} \mathrm{~s}^{-2}$ ) pressure (blue lines ci $=6 \mathrm{hPa}$ ), and $\theta$ (red lines, $\mathrm{ci}=5 \mathrm{~K})$ at $7 \mathrm{~km}$ at the same times as GWs are shown in Fig. 15 . Timings are also summarized in Table 2 . The distance between tick marks is $300 \mathrm{~km}$.

$24 \triangle N B E$ anomalies (blue lines, ci $=0.01 \times 10^{-8} \mathrm{~s}^{-2}$ ) and $\theta$ (red lines, $\mathrm{ci}=5 \mathrm{~K}$ ) along cross sections indicated in Fig. 15. 


\section{INTRODUCTION}

Gravity waves are one of the most fundamental dynamical processes in the atmosphere. They are closely associated with a wide variety of atmospheric processes, ranging from microscale to synoptic scale dynamical phenomena, such as clear air turbulence, convection and baroclinic waves. Generally, gravity waves (GWs) can be generated by topography, frontogenesis, convection, shear instability, geostrophic adjustment and wave-wave interactions (Koch and Dorian 1988; Fritts and Alexander 2003).

Typical mesoscale gravity waves have horizontal wavelengths of $50-500 \mathrm{~km}$, vertical wavelengths of 1-4 km, periods of $0.5-4 \mathrm{~h}$, amplitudes of $0.5-15 \mathrm{hPa}$ (pressure perturbations at surface) and phase velocities of $15-35 \mathrm{~m} / \mathrm{s}$. Previous studies (Uccellini and Koch 1987, hereinafter UK87; O'Sullivan and Dunkerton 1995, hereinafter OD95; Bosart et al. 1998; Zhang et al. 2001; Zhang 2004, hereinafter Z04) have shown that inertia-gravity waves can be generated in the vicinity of upper level jet streaks during life cycles of baroclinic waves. These past studies have identified geostrophic adjustment as a principal mechanism for gravity wave generation in the upper-level jet streaks.

The atmosphere is continuously adjusting. The result of different types of adjustment, including geostrophic adjustment (Rossby 1937; Cahn 1945), baroclinic adjustment (Stone 1978) and hydrostatic adjustment (Bannon 1995), is that the energy associated with imbalance is emitted such that the atmosphere is able to maintain a quasibalanced state as one observes. Any adjustment thus involves an energy transfer process.

This thesis follows the style of Journal of Atmospheric Sciences. 
For geostrophic adjustment, dispersive gravity waves contribute to a radiation of excessive energy, which yields redistribution of mean momentum, energy and potential vorticity allowing the flow to regain a geostrophically balanced state (Cahn 1945; Blumen 1972; Kaplan et al. 1997).

In addition, earlier case studies with observational data also revealed a significant impact of mesoscale gravity waves on surface wind, cloud cover and precipitation (e.g. Koch and O’Handley 1997). These studies attributed the excitation mechanisms to various sources, such as fronts, topography, convection, shear instability and geostrophic adjustment. Further complications of wave generation arise in the context of background baroclinic waves. Mid-latitude baroclinic waves, developed from initial baroclinic instability, may lead to various synoptic-scale processes, such as cyclones and anticyclones, surface fronts and upper level jet-fronts (Pierrehumbert and Swanson 1995). UK87 summarized 13 case studies of gravity waves concluding that a common synopticscale baroclinic environment conducive for generation and maintenance of gravity waves is a region bounded by the jet axis to the west or northwest, a surface front to the southeast, an inflection axis to the southwest and a $300 \mathrm{hPa}$ ridge axis to the northeast. Shearing instability and geostrophic adjustment are considered as two potential source mechanisms, owing to the existence of jet streaks near the region of observed waves. The GW wavelengths predicted by the shear instability mechanism are much shorter than those of commonly observed GWs. This discrepancy suggests geostrophic adjustment as the only major source mechanism of mesoscale gravity waves in the typical environment of UK87. However, due to the fact that surface features and upper-level front jet are integral parts of baroclinic waves, exact roles of individual components on GWs are hard 
to assess. By using high-resolution sounding data, Guest et al. (2000) also identified a similar synoptic pattern common for inertia gravity waves in the lower stratosphere. The rotary spectrum and hodograph analysis showed dominance of upward energy propagation in the stratosphere and downward energy propagation in the troposphere, which suggests that GWs are generated near the tropopause. Their ray-tracing results lent further support to the hypothesis that these GWs are indeed generated in regions of upper-tropospheric jet-front systems.

Comprehensive studies such as Bosart et al. (1998) benefit from a wide array of data sources such as surface observations, radar and satellite images, and radiosondes. However, GWs of mesoscale nature are not easily detected by the observational network. Case studies using conventional observations suffer from limited data availability in time and space. Even in cases of luckily observed GWs, it is often difficult to identify all the wave parameters and source mechanisms.

Consequently, the ambiguous connection between gravity waves and the background baroclinic environment inspires interest in numerical investigations of gravity waves. One advantage of using numerical models is that it is easy to obtain fine structures of mesoscale systems.

OD95 made a first attempt to simulate gravity waves initiated from idealized baroclinic waves with a $3 \mathrm{D}$, hydrostatic primitive equation model and to investigate wave generation by geostrophic adjustment. Sub-synoptic scale gravity waves with horizontal wavelengths of $600-1000 \mathrm{~km}$ in the jet exit region were successfully produced in their simulations. However, the horizontal grid spacing used in their semi-global models was only approximately $50 \mathrm{~km}$, which is too large to resolve typical mesoscale gravity waves 
(horizontal wavelengths 50-500 km, eg. UK87; Zhang and Koch 2001; Zhang et al. 2001; Koch et al. 2001). The Lagrangian Rossby number combined with the mean flow kinetic energy was employed as the indicator of geostrophic imbalance to demonstrate that strong radiation by gravity waves is present in regions of imbalance. Zhang (2004, Z04) performed idealized baroclinic wave simulations using a high-resolution mesoscale model with horizontal grid spacing as small as $3.3 \mathrm{~km}$. The mesoscale gravity waves produced in the constant f-plane model used by Z04 had prevalent horizontal wavelengths of 100-200 km and a frequency of approximately 3-4 times of the Coriolis parameter $\mathrm{f}$ in association with an upper level jet-front system. The vertical and horizontal wavelengths and frequencies were interrelated, conforming to the linear gravity wave dispersion relation (Gill 1982).

At the mature stage of baroclinic waves, geostrophic balance breaks down where jets are distorted and nonlinear effects take over (OD95, Hakim 2000). Therefore, it is beneficial to investigate the nonlinear effect near a jet-front system. The nonlinear balance equation (Charney 1952) may serves as an ideal tool for this purpose. Indeed, it has been used in a number of studies. For example, Raymond (1992) demonstrated the effectiveness of nonlinear balance for mesoscale processes for Rossby around unity.

A mesoscale gravity wave is in itself highly unbalanced. Regions of non-zero residue of the nonlinear balance equation

$$
\Delta N B E=2 J(u, v)+f \zeta-\alpha \nabla^{2} P
$$

are unbalanced even in terms of high-level nonlinear balance. $\triangle N B E$ was shown to be more accurate than other imbalance diagnosis tools (Zhang et al. 2000) and was used by Z04 to explore the flow imbalance. Z04 illustrated that flow imbalance is excited 
continuously by the distorted jet streaks. Wave signals in the divergence field follow the local non-zero $\triangle N B E$ occurrence. The spontaneous gravity wave emissions counteract the production of imbalance, acting to restore the balance. This transient process, called balance adjustment as a generalization of geostrophic adjustment, was hypothesized as the mechanism of gravity wave generation (Z04). Flow imbalance diagnosis in this stage can be expected to provide further insight into the theory of balance adjustment.

The observational studies together with idealized simulations have demonstrated that mesoscale gravity waves can be generated from background baroclinic waves. In this investigation, we will conduct various sensitivity tests based on Z04 in the study of gravity wave characteristics and source mechanisms, which are impossible to accomplish with the standard or even enhanced observations (Powers and Reed 1993). In theory, characteristics of gravity waves are determined by their sources and propagation media. The study of sensitivities of embedded GWs requires well developed baroclinic waves. In the current study, the jet strength and tropospheric static stability are treated as determinative factors to alter the baroclinic growth rate (Lindzen and Farrell, 1980). The relation between the characteristics, distribution and variability of the gravity waves and their parent baroclinic jet-front systems will be investigated, complementary to Z04.

The aim of this study is to examine the generation of gravity waves during different life cycles of idealized baroclinic waves. We intend to extend Z04 in two major aspects:

- Explore the dynamic relationship between the gravity waves and the evolution of background baroclinic jet-front systems.

- Test the sensitivity of wave parameters to the baroclinic wave development. 
Methods and experimental design will be described in the next section. Z04 will be revisited in the third section, in addition to discussion on diagnostics of flow imbalance and its surrounding mesoscale environments. Section 4 will provide an overview of all experiments, followed by detailed comparison. Further discussion on flow imbalance and balance adjustment will be presented in section 5. Summary and implications in section 6 will conclude this study. 


\section{METHODS}

\subsection{Initial conditions}

As in Z04, the mesoscale model used in this study is configured to eliminate the effects of spherical geometry, non-uniform Coriolis parameter, topography and moist processes. The potential vorticity inversion technique (Davis and Emanuel 1991) is first employed to create a 2-D idealized baroclinic jet similar to Simmons and Hoskins (1978), which is then expanded to a 3-D grid assuming zonal homogeneity. The 3-D PV distribution with addition of a PV perturbation at the tropopause level is then inverted to produce the initial conditions for the coarse MM5 domain (Z04). This upper level disturbance along the center of the jet streak is shown in Fig. 1, which initiates the development of an extratropical surface cyclone. Fixed lateral boundary conditions are applied in all directions in the coarse simulation. By making the model domains in all directions sufficiently large, baroclinic wave life cycles can be produced with negligible influence from lateral boundaries. The nesting capability of the MM5 system is then applied to allow multiple two-way nested domains with horizontal grid spacing sufficiently small to resolve mesoscale gravity waves.

Steps to create the initial condition are similar to Z04. We will describe the procedures in detail, providing an outline of how various initial conditions are created. The first step is to create a two dimensional PV distribution and invert the velocity and $\theta$ fields. A 2D Ertel Potential Vorticity (EPV) inversion under zonal homogeneity assumption is:

$$
\Phi_{y y}=f \Psi_{y y}
$$




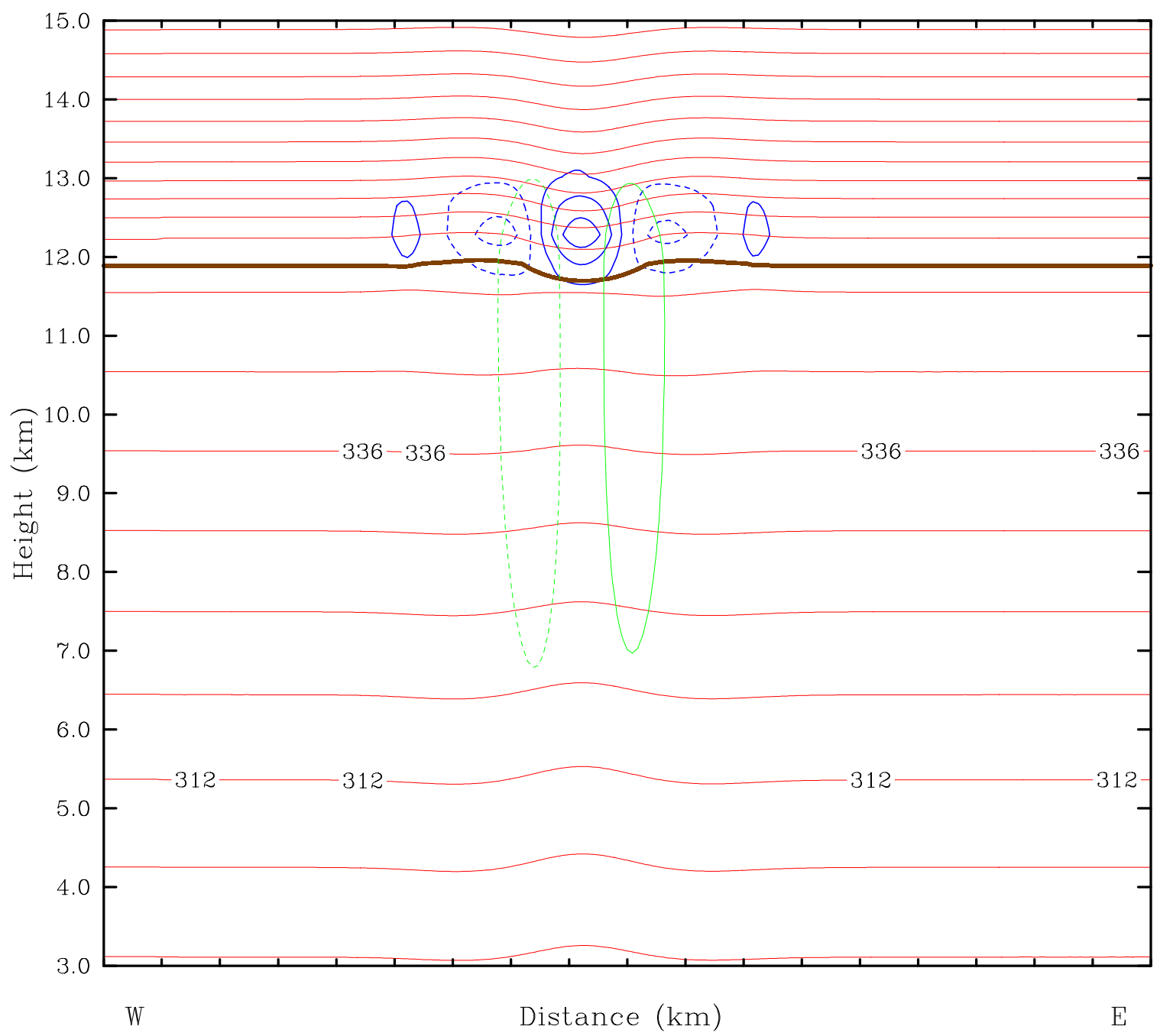

Figure 1: Initial conditions of CNTL from west to east in the middle of Domain 1. PV anomalies (blue thin lines, $\mathrm{ci}=0.1 \mathrm{PVU}$, negative value dashed), meridional winds $(\mathrm{ci}=2.5 \mathrm{~m} / \mathrm{s})$ green thin lines, negative value dashed), $1.5 \mathrm{PVU}$ potential vorticity (brown thick line) and potential temperatures (red thin lines, $c i=6 \mathrm{~K}$ ). The distance between tick marks is $1000 \mathrm{~km}$. 


$$
P V=-\frac{g \kappa \pi}{p}\left[\left(f+\Psi_{y y}\right) \Phi_{\pi \pi}-\Psi_{y \pi} \Phi_{y \pi}\right]
$$

where Exner function $\pi$ is taken as the vertical coordinate. $\Psi, \Phi$ and PV represent stream function, geopotential and Ertel potential vorticity respectively.

Following RSS94, PV in the troposphere and stratosphere is assigned to be a constant value, e.g., in CNTL simulations (Z04), $P V_{T}=0.5 \times 10^{-6} P V U$ and $P V_{S}=4.0 \times 10^{-6} P V U$. A relatively small $P V_{T}$ represents low static stability in the troposphere. We will use various $P V_{T}$ ranging from $0.25-0.5$ PVU to represent the troposphere with varying initial static stability. PV in the domain $\left(p_{r} \in(10 h p a, 1000 h p a), y \in\left(0, y_{L}\right), y_{L}=9000 \mathrm{~km}\right)$ can be specified as

$$
P V=\frac{P V_{T}+P V_{S}}{2}-\frac{P V_{S}-P V_{T}}{2} \cdot \tanh \left(\frac{2.65 \times\left(p_{r}-P_{T}\right)}{\Delta p_{t r}}\right)
$$

$p_{r}$ is the reference pressure at each level. $\Delta p_{t r}$ is $15 \mathrm{hPa}$ giving the thickness of the tropopause. The pressure of the flexible tropopause $P_{T}$ is given by

$$
\begin{aligned}
P_{T}=p m_{t p}- & \Delta p_{e} \cdot \tanh \left(\frac{2.65 \times\left(y-y_{L} / 2\right)}{\Delta y_{e}}\right)+ \\
& 1.25 \times \Delta p_{e s} \cdot \tanh \left(\frac{2.65 \times\left(y-y_{L} / 4\right)}{2 \Delta y_{e}}\right)+0.75 \times \Delta p_{e s} \cdot \tanh \left(\frac{2.65 \times\left(y-3 y_{L} / 4\right)}{2 \Delta y_{e}}\right)
\end{aligned}
$$

where $\mathrm{pm}_{t p}$ is the reference pressure of the tropopause $(300 \mathrm{hPa}) ; \Delta y_{e}$ is the horizontal scale of the sloping tropopause $(600 \mathrm{~km}) ; \Delta p_{e}$ is the maximum departure in pressure of the tropopause in the northern and southern portions of jet from its average level; $\Delta p_{e s}$ is the displacement of the tropopause in pressure near the northern and southern boundaries where the velocity is close to zero. In CNTL, $\Delta p_{e}$ and $\Delta p_{e s}$ are $112.5 \mathrm{hPa}$ and $30 \mathrm{hPa}$ 
respectively. Increasing $\Delta p_{e}$ and $\Delta p_{e s}$ leads to a stronger jet, i.e., stronger horizontal and vertical shear. A stronger jet is given by $\Delta p_{e}=135 \mathrm{hPa}$ and $\Delta p_{e s}=35 \mathrm{hPa}$. Decreasing $\Delta p_{e}$ and $\Delta p_{e s}$ leads to a weaker jet, i.e., weaker horizontal and vertical shear. A weaker jet is given by $\Delta p_{e}=90 \mathrm{hPa}$ and $\Delta p_{e s}=25 \mathrm{hPa}$. These three configurations of $\Delta p_{e}$ and $\Delta p_{e s}$ are repeatedly used in creating modified jet strength experiments.

Top boundaries are set as constant temperature $600 \mathrm{~K}$. At the southern and northern boundaries vorticity is assumed to be zero. Integrating PV ( $P V=f \partial \theta / \partial p$ ) from top to bottom gives temperature at lateral boundaries. The bottom boundary conditions are linearly varying geopotential heights.

The second step is to expand the $2 \mathrm{D}$ jet streak (in the zonal direction) to derived the three-dimensional fields and then add a localized 3D PV perturbation near the tropopause. Before performing the 3D EPV inversion, we add an initial PV perturbation by displacing the tropopause northward following Rotunno and Bao (1996). The PV perturbation is given by

$$
P V^{\prime}(x, y, \pi)=\frac{\partial P V}{\partial y} \cdot \sin \left(\frac{\pi\left(y-y_{c}\right)}{y_{S L}}\right) \cdot \cos \left(\frac{2 \pi\left(x-x_{c}\right)}{x_{S L}}\right) \cdot \frac{\left(-0.3 \times 10^{6}\right) \cdot a^{2}}{a^{2}+\left(x-x_{c}\right)^{2}}
$$

for $\mathrm{x} \in(5000 \mathrm{~km}, 11000 \mathrm{~km}), \mathrm{y} \in(3000 \mathrm{~km}, 5000 \mathrm{~km})$. Other parameters are specified as $\mathrm{x}_{\mathrm{SL}}=4000 \mathrm{~km}, \mathrm{y}_{\mathrm{SL}}=8000 \mathrm{~km}, \mathrm{x}_{\mathrm{c}}=8000 \mathrm{~km}, \mathrm{y}_{\mathrm{c}}=0$, and $\mathrm{a}=1.863 \times 10^{6} \mathrm{~m}$. Fig. 1 shows the initial PV perturbations, potential temperature and associated meridional wind field in a west east cross section at the center of the domain.

A 3D Ertel PV inversion system has governing equations (Davis and Emanuel 1991)

$$
\nabla^{2} \Phi=\nabla(f \nabla \Psi)+2\left(\Psi_{x x} \Psi_{y y}-\Psi_{x y}^{2}\right)
$$




$$
P V=-\frac{g \kappa \pi}{p}\left[\left(f+\nabla^{2} \Psi\right) \Phi_{\pi \pi}-\Psi_{x \pi} \Phi_{x \pi}-\Psi_{y \pi} \Phi_{y \pi}\right]
$$

Bottom and lateral boundary conditions are potential temperature determined from the 2D PV inversion. The final step is to invert the 3D PV including localized PV perturbations to obtain initial conditions for the MM5 system.

In summary, the strength and geometry of the jet streaks and the potential vorticity are all specified and adjustable in the initial conditions. These modifications then change horizontal and vertical shear and static stability directly or indirectly. By tuning these large-scale flow parameters, the growth rate of baroclinic instability is changed, which consequently enables us to design a series of sensitivity experiments to explore the sensitivity of gravity waves to the background baroclinic waves.

\subsection{Experimental design}

To investigate the sensitivity of GWs to background baroclinicity, various jetfront systems are needed to provide different large-scale flow patterns which can generate GWs. This is achieved by modifying two parameters in the initial conditions. The first step is to generate a set of BWs with different characteristics, which can be achieved by modifying the growth rate of BWs. The growth rate of the fastest growing normal mode for BWs in the simple Charney model is given by a baroclinicity index,

$$
\sigma=0.31 \cdot \frac{f}{N}\left|\frac{\partial u}{\partial z}\right|
$$

stating that Coriolis parameter, $f$, static stability, $N^{2}$, and average zonal wind shear $\left|\frac{\partial u}{\partial z}\right|$ are important for baroclinic instabilities (Lindzen and Farrell 1980). In addition to 
the CNTL of Z04, four more high-resolution experiments are conducted through modifications of the slope of the tropopause and/or tropospheric PV (shear and static stability) in deriving the initial conditions for the control simulation (same as in Z04). A steeper tropopause and a lower tropospheric PV will lead to stronger vertical shear and lower stability.

Three of the sensitivity experiments (BigS, LowS and BigN) are designed to have different baroclinic growth rate from CNTL through changing either the slope of the tropopause or the tropospheric PV in the initial conditions in CNTL which should leads to a relatively faster or slower baroclinic growth rate. The other experiment ("LowSlowN") changes the initial shape of the tropopause and tropospheric PV simultaneously to obtain a BW growth rate in terms of minimum SLP (sea level pressure) similar to the control simulation. Planetary rotation is fixed to be the same as CNTL for all experiments. Sensitivity experiments on change of Coriolis parameter will be discussed elsewhere.

The parameters used to initiate the five experiments including CNTL examined in Z04 are summarized in Table 1. Figure 2 shows the unperturbed initial conditions of all five experiments in meridional cross sections, including potential temperature, wind speed and dynamic tropopause, defined as 1.5 PVU. A relatively flat tropopause attends in all the cases involving an initially low-shear tropopause. A relatively steep tropopause attends in all cases involving strong shear. Maximum zonal wind speed shown in Fig. 2 is also summarized in Table 1. 
Table 1: Summary of the five experiments. The first column is experiments. The second and third columns are the tropopause geometry parameters. The fourth column is $P V_{T}$ used in the 2D inversion. The fifth column is the maximum zonal wind speed. The sixth and seventh columns are start times of Domains 2 and 3. The last column is the total integration time.

\begin{tabular}{|c|c|c|c|c|c|c|c|}
\hline \multirow{2}{*}{ EXPR } & \multicolumn{2}{|c|}{$\begin{array}{c}\text { Tropopause } \\
\text { Geometry }\end{array}$} & \multirow{2}{*}{$\begin{array}{c}P V_{T} \\
(\mathrm{PVU})\end{array}$} & $\begin{array}{c}U_{\max } \\
(\mathrm{m} / \mathrm{s})\end{array}$ & $\begin{array}{c}\mathrm{D} 2 \\
\text { (hour) }\end{array}$ & $\begin{array}{c}\text { D3 } \\
\text { (hour) }\end{array}$ & $\begin{array}{c}\text { Total } \\
\text { integrati } \\
\text { on time } \\
\text { (hours) }\end{array}$ \\
\cline { 2 - 7 } CNTL & 112.5 & 30 & 0.5 & 46.7 & 72 & 84 & 120 \\
\hline BigS & 135 & 35 & 0.5 & 53.7 & 48 & 60 & 96 \\
\hline LowS & 90 & 25 & 0.5 & 39.2 & 108 & 96 & 144 \\
\hline LowN & 112.5 & 30 & 0.25 & 56.4 & 30 & 36 & 66 \\
\hline LowNlowS & 90 & 25 & 0.46 & 38.3 & 60 & 72 & 120 \\
\hline
\end{tabular}



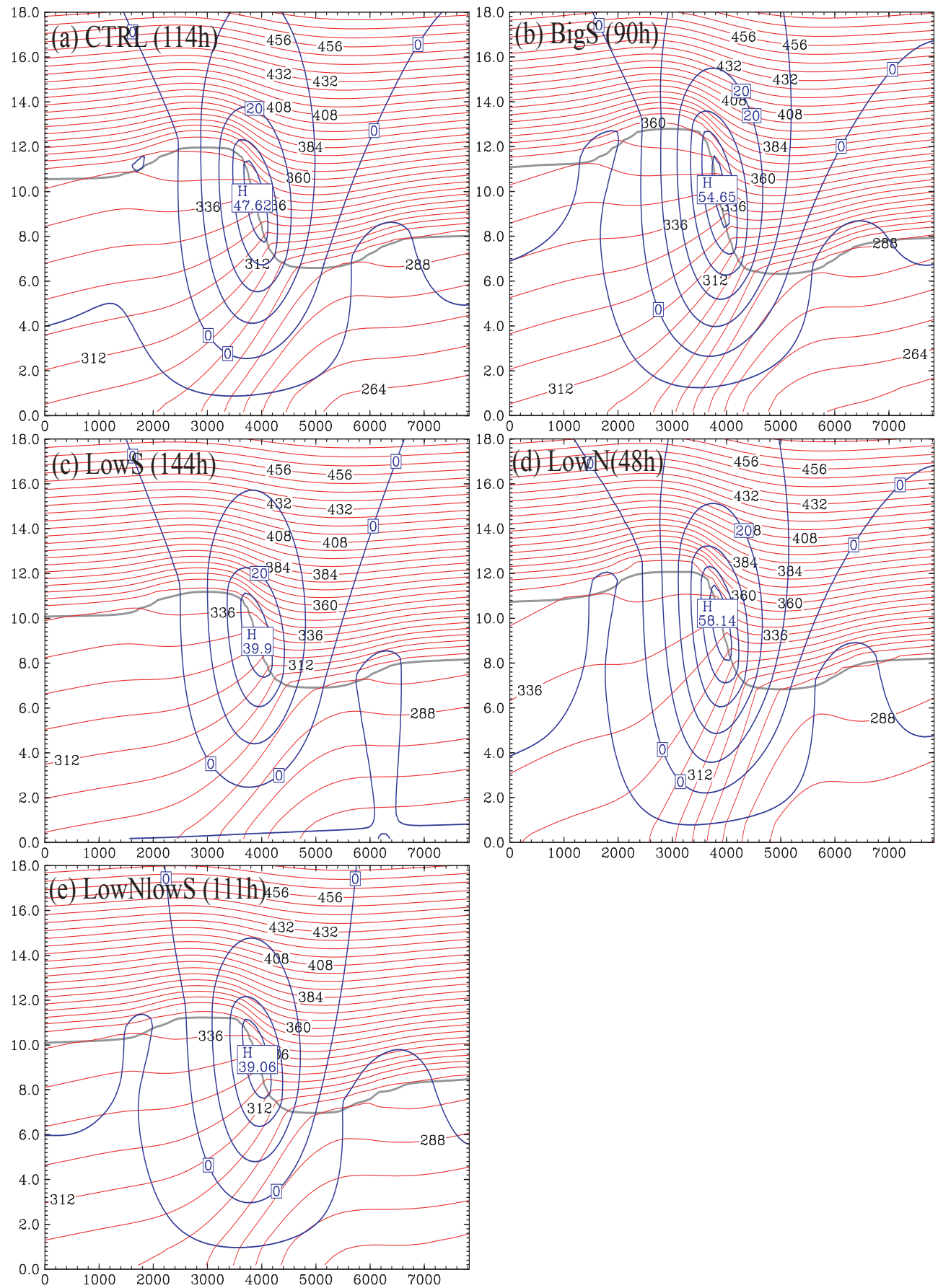

Figure 2: Initial conditions for 5 experiments. Vertical cross section of initial potential temperature (thin line, $\mathrm{ci}=6 \mathrm{~K}$ ) and zonal velocity (thick line, $\mathrm{ci}=10 \mathrm{~m} \mathrm{~s}^{-1}$ ) for the initial basic-state jet. The gray thick line denotes the location of the tropopause where potential vorticity equals $1.5 \mathrm{PVU}$.) 


\section{CONTROL SIMULATION AND Z04 REVISITED}

In CNTL, the early development of dry BWs can be described by the linear theory of baroclinic instability. After 72h, surface features in common with well-developed BWs include a continuously deepening surface cyclone and anticyclone, strengthening cold and warm fronts, and emergence of an occluded front as part of a "T bone" structure. The tropopause above the surface cyclone descends continuously accompanied by a strengthening upper level jet.

Around $78 \mathrm{~h}$, the BW matures to a point where the quasi-linear growth stage ends (see section 4a). Around this time, an area of imbalance with $\triangle N B E$ greater than $0.5 \times 10^{-9} \mathrm{~s}^{-2}$ begins to be pronounced near the tropopause (Figs. 10- 11 in Z04) denoted by the $1.5 \mathrm{PVU}$ contour. The GW signals in the exit region of upper level jet streak starts to emerge 24 hours later, i.e. 102h (Z04 Fig. 11d), in the $\triangle N B E$ field in the vertical plane. These GW signals are barely noticeable in the vertical velocity fields at this time (Fig. 5 in Z04). At 114h and 120h, vertical motions show strong wave signals in the horizontal and vertical planes (Fig. 3).

As described in section 2.1, a noteworthy feature of the initial 2D jet and the 3D disturbance is that they are both nonlinearly balanced. Flow imbalance is nevertheless produced during the life cycles of BWs in the high-resolution primitive models. The imbalance diagnosis in Z04 leads to a high-level balance adjustment hypothesis as an extension of the classical geostrophic adjustment theory. Nonlinear balance is believed to be an appropriate assumption with moderate accuracy on broad scales of motions, even on a broad range of scales with a large Rossby number (e.g., Raymond 1992, Zhang et al. 2000). Allen (1991) demonstrated that nonlinear balance is the minimum energy state for 

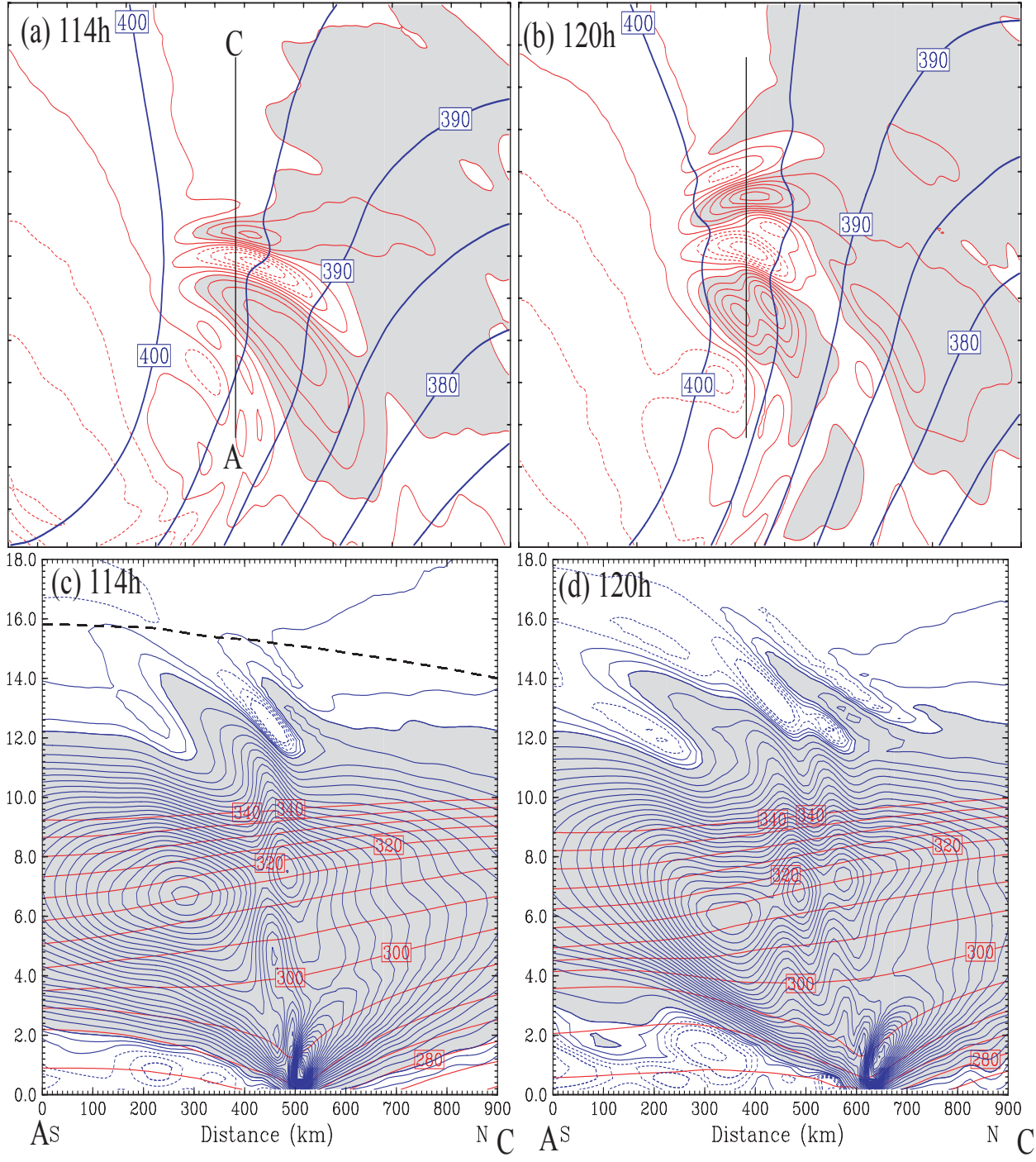

Figure 3: Vertical velocities adapted from Fig. 6 in Z04. CNTL simulated potential temperature (thick line, $\mathrm{ci}=5 \mathrm{~K}$ ) and vertical velocity (solid, positive; dashed, negative; $\mathrm{ci}=2 \times 10^{-3} \mathrm{~m} / \mathrm{s}$; values $>5 \times 10^{-3} \mathrm{~m} / \mathrm{s}$ shaded) at the $13-\mathrm{km}$ level valid at (a) 102 and (b) $108 \mathrm{~h}$, and along the cross section $\mathrm{AB}$ valid at (c) 102 and (d) $108 \mathrm{~h}$. The straight line $\mathrm{AB}$ in (a) and (b) denotes the location of the cross section in (c) and (d). Contours of potential temperature greater than $340 \mathrm{~K}$ are generally flat but dense in the stratosphere and were thus not plotted in (c) and (d). The distance between tick marks in (a) and (b) is $100 \mathrm{~km}$. 
the shallow water system. The extent of imbalance away from nonlinear balance can be conveniently evaluated by the residual of the nonlinear balance equation. In contrast, the ageostrophic wind and Rossby number are merely defined as deviations from geostrophy, which is the reason variants of Rossby number are needed in addition to the Eulerian Rossby number (see recent review by Zhang et al. 2000). The balance adjustment hypothesis, similar to canonical geostrophic adjustment theory, involves the generation of gravity waves in favor of restoration of a realizable balance state.

However, this imbalance does not diminish even after GWs propagate away from the jet to high levels, e.g. $13 \mathrm{~km}$. Z04 demonstrated that localized imbalance during $78 \mathrm{~h}-$ 108h in Fig. 4 a-f (Fig. 11 in Z04) actually increases substantially, contrary to its expected reduction by the canonical geostrophic adjustment processes. This counterintuitive development of imbalance can be reconciled provided that the continuous imbalance produced by large scale dynamics overtakes the amount of decrease in imbalance by adjustment though emission of GWs (Z04).

To facilitate better understanding of the balance adjustment hypothesis developed in $\mathrm{Z} 04$, and identify the origin of the imbalance in terms of $\triangle N B E$, the mesoscale details of the jet front systems are further examined.

Going back to previous studies, a comprehensive review of upper level frontogenesis and associated secondary circulation concluded tilting to be major contributor to upper-level frontogenesis (Keyser and Shapiro 1986). Rotunno et al. (1994; referred as RSS94 hereinafter) and Wandishin et al. (2000) performed an analysis of 3D frontogenesis processes in a primitive equation model. These attempts were made respectively from two different perspectives: traditional QG analysis, i.e., geostrophic 

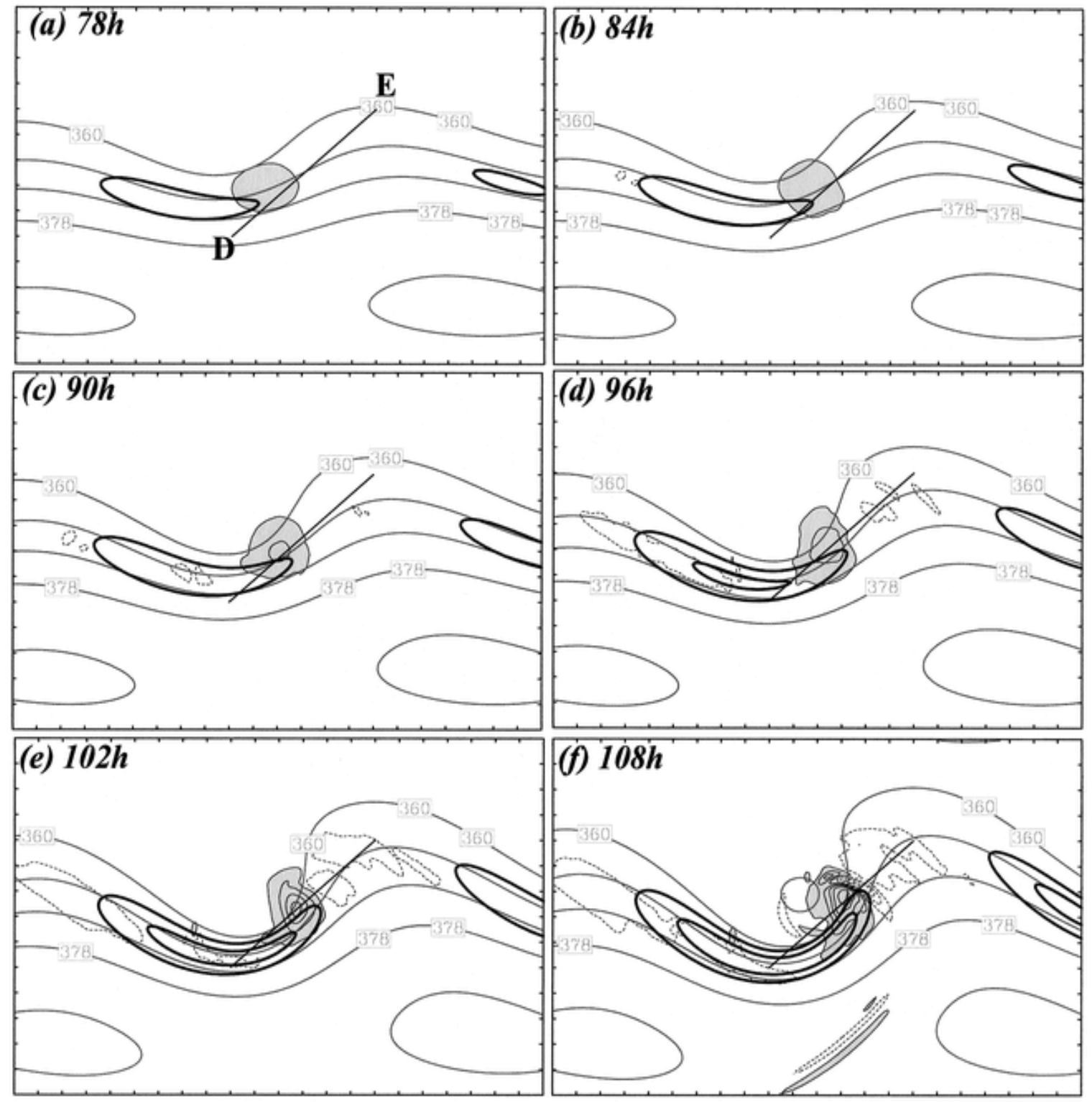

Figure 4: CNTL simulated 8-km pressure (thick line, $\mathrm{ci}=4 \mathrm{hPa}$ ), the residual of the nonlinear balance equation (shaded, solid, positive; dashed, negative; ci=1 $1 \times 10^{-9} \mathrm{~s}^{-2}$ ), and wind speed over $40 \mathrm{~m} / \mathrm{s}$ (bold contours, ci=5m/s) valid at (a) 78, (b) 84, (c) 90, (d) 96 , (e) 102 , and (f) $108 \mathrm{~h}$ The straight line DE denotes the location of the cross section plotted in Fig. 11 . The distance between tick marks is $300 \mathrm{~km}$. Adapted from Fig. 9 in Z04. 
and ageostrophic winds inferred from the Q vector (RSS94); and a PV perspective by piecewise static QGPV inversion technique (Wandishin et al. 2000). In the PV perspective, vertical shear inferred from cold advection was stressed for its contribution to foldogenesis. Foldogensis diagnoised on a PV plane has an equivalent role as conventional frontogenesis (Wandishin et al. 2000).

Fig. 5, adapted from Fig. 13 in RSS94, shows the evolution of potential temperature $(\theta)$, geopotential $(\phi)$ and 1.5 PVU from day 6 to day 9 . The phase lag of the synoptic temperature wave with regard to the geopotential wave remains very small at 6 $\mathrm{km}$ (the level of upper level frontogenesis) around day 6 (Fig. 5a). This pattern changes around day 7 (Fig. 5b), after which the angle between isotherms and constant $\phi$ lines becomes increasingly larger (Fig $5 \mathrm{c}$ and $\mathrm{d}$ ). The northward turning of isotherms upstream of the trough is associated with high PV extrusion into the $6 \mathrm{~km}$ level.

The initial conditions in CNTL are nearly identical to RSS94 in terms of basic state jet structure, both of which are created following the model of "constant-potential vorticity troposphere with constant-potential-vorticity stratosphere(RSS94)". Consequently, a similar BW was produced by the MM5 simulation (Z04). Mesoscale GWs near the surface front and the upper level jet are clearly established in the high resolution model. In contrast, no GW signals were identified in their coarse model with a horizontal grid spacing of 100km by RSS94. Fig. 6 shows CNTL results including the pressure (long dashed), potential temperature (solid), and overlapped by tropopause (thick solid). In CNTL, the gradually increasing angle between $\theta$ contours and $\phi$ contours at $6 \mathrm{~km}$ is reproduced much earlier due to the faster growth of the amplifying BW. 
(a) day 6

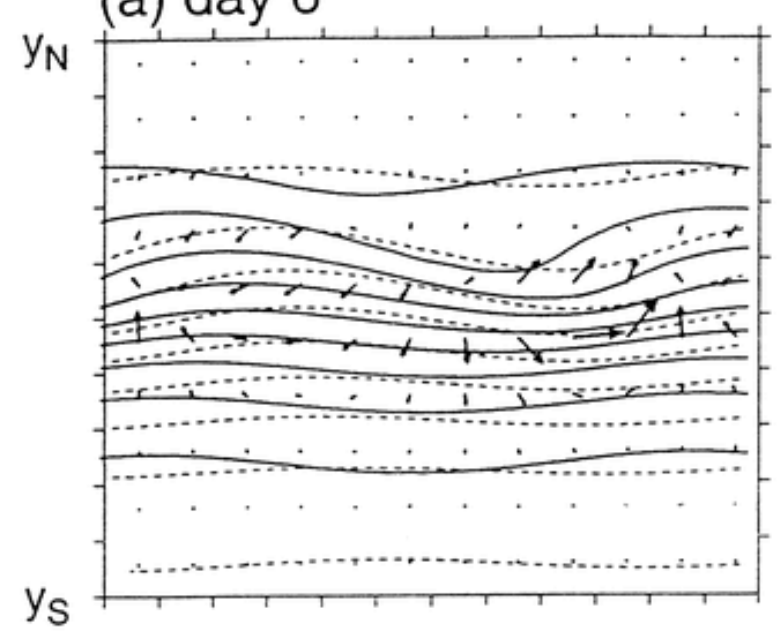

(c) day 8

$\mathrm{y}_{\mathrm{N}}$

$\mathrm{y}_{\mathrm{S}}$

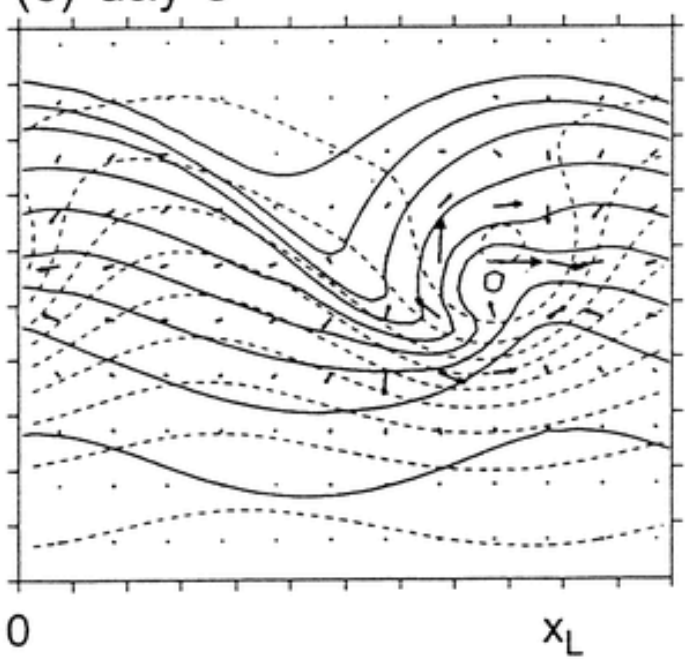

(b) day 7
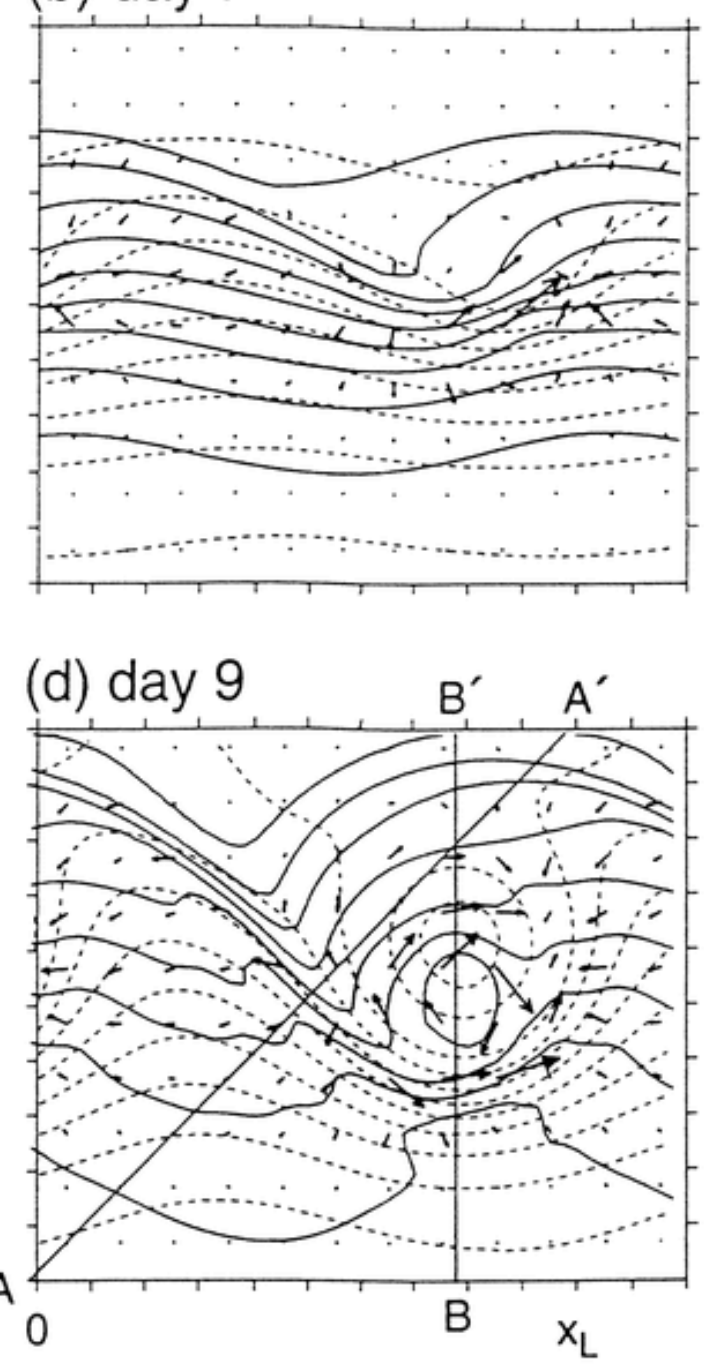

Figure 5: Potential temperature $\theta(\mathrm{ci}=5 \mathrm{~K}), Q$ vector and geopotential $\phi$ $\left(\mathrm{ci}=1000 \mathrm{~m}^{2} \mathrm{~s}^{2}\right)$ at $7 \mathrm{~km}$. Adapted from RSS94 Fig. 13. 


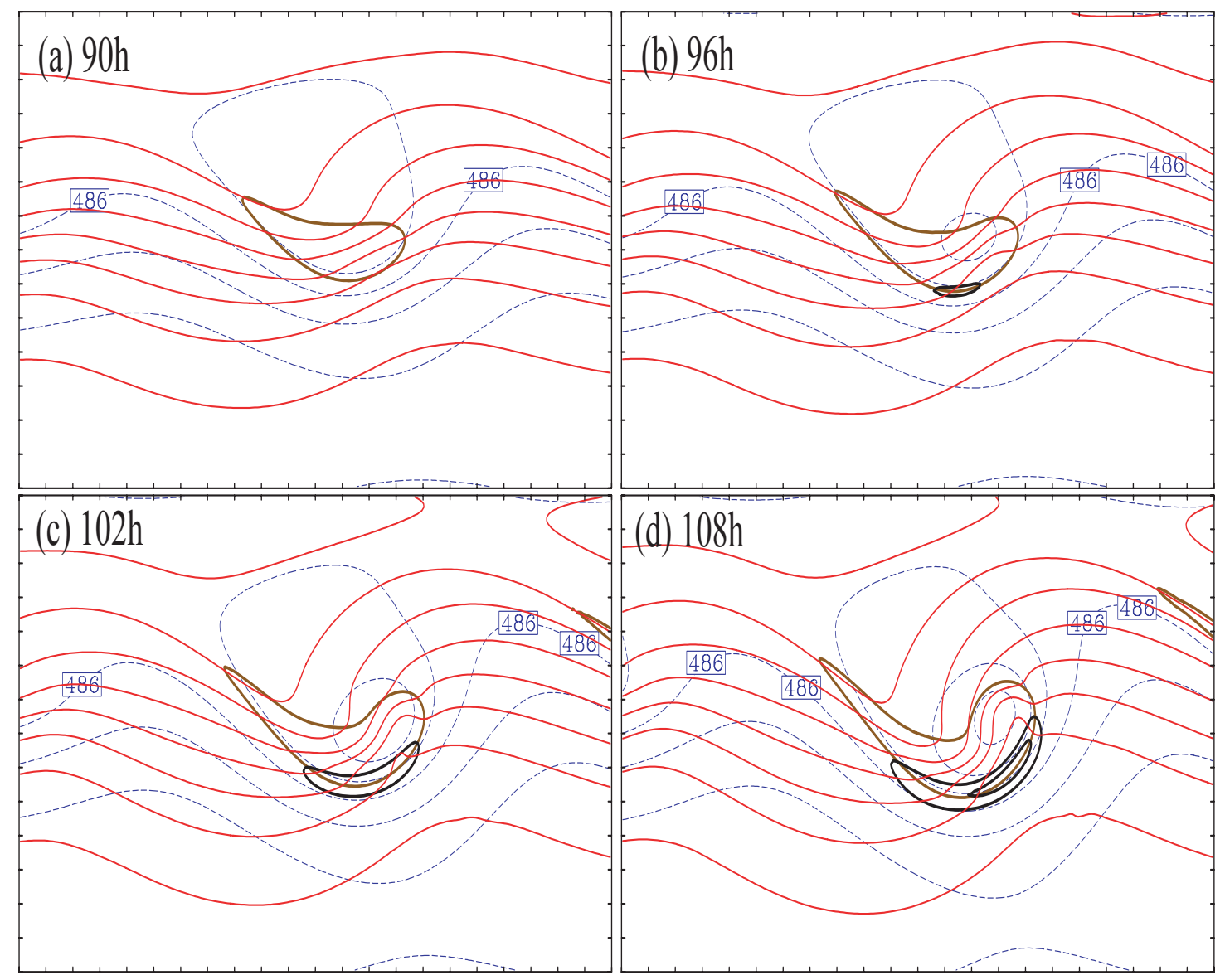

Figure 6: CNTL simulated flow pattern at 6km at 90h (a), 96h(b), 102h(c), 108h(d). Pressure (dashed lines, $\mathrm{ci}=6 \mathrm{hPa}$ ), potential temperature $\theta$ (red solid lines, ci $=5 \mathrm{~K}$ ), 1.5 PVU (brown thick lines) and wind speed (black lines, ci $=5 \mathrm{~m} / \mathrm{s}$ starts at $40 \mathrm{~m} / \mathrm{s}$ ). The distance between tick marks is $300 \mathrm{~km}$. 
Fig. 7 shows the pattern of 1.5 PVU (thin solid lines), vertical velocities (positive values by thick solid lines and negative values by dash lines) and $\triangle N B E$ (thick lines and short dashed, shaded regions). At 96h, warm air from the stratosphere appears to intrude downward forming the temperature ridge and intense thermal gradient, representing the dramatic pattern change mentioned in RSS94. Regions of downward motions expand downstream from $96 \mathrm{~h}$ to $108 \mathrm{~h}$. At the edge between regions of upward and downward motions, $\triangle N B E$ are consistently enhanced. It is noted that the imbalance regions collocate with pattern changes on isotherms north of the pressure trough, as well as the regions of maximum gradient of vertical motions. The horizontal divergence field bears nearly the same features as the vertical motions (not shown). Regions of maximum $\triangle N B E$ again coincide with maximum gradient of divergence.

In a PV perspective, upper level jet fronts are manifested as tropopause folding (Wandishin et al. 2000). Fig. 8 shows $\triangle N B E$ and geopotential height on the tropopause, defined as 1.5 PVU. Steep geopotential gradients ahead of localized $\triangle N B E$ in trough regions of PV waves strengthen from $90 \mathrm{~h}$ to $108 \mathrm{~h}$. A new localized $\triangle N B E$ maximum also develops a whole BW wavelength upstream of the major imbalance. As Zhang et al. (2000) discussed, an unbalanced perturbation pressure field can be obtained by subtracting the balanced pressure field from the original pressure field. The balanced pressure was computed through 3D PV inversion. The imbalance in terms of perturbation pressure field has a similar pattern to that of $\triangle N B E$. In summary, imbalance indicated by residuals of nonlinear balance equation collocate with regions of unbalanced pressure, maximum gradient of divergence and vertical motions near the upper level jet front systems. 


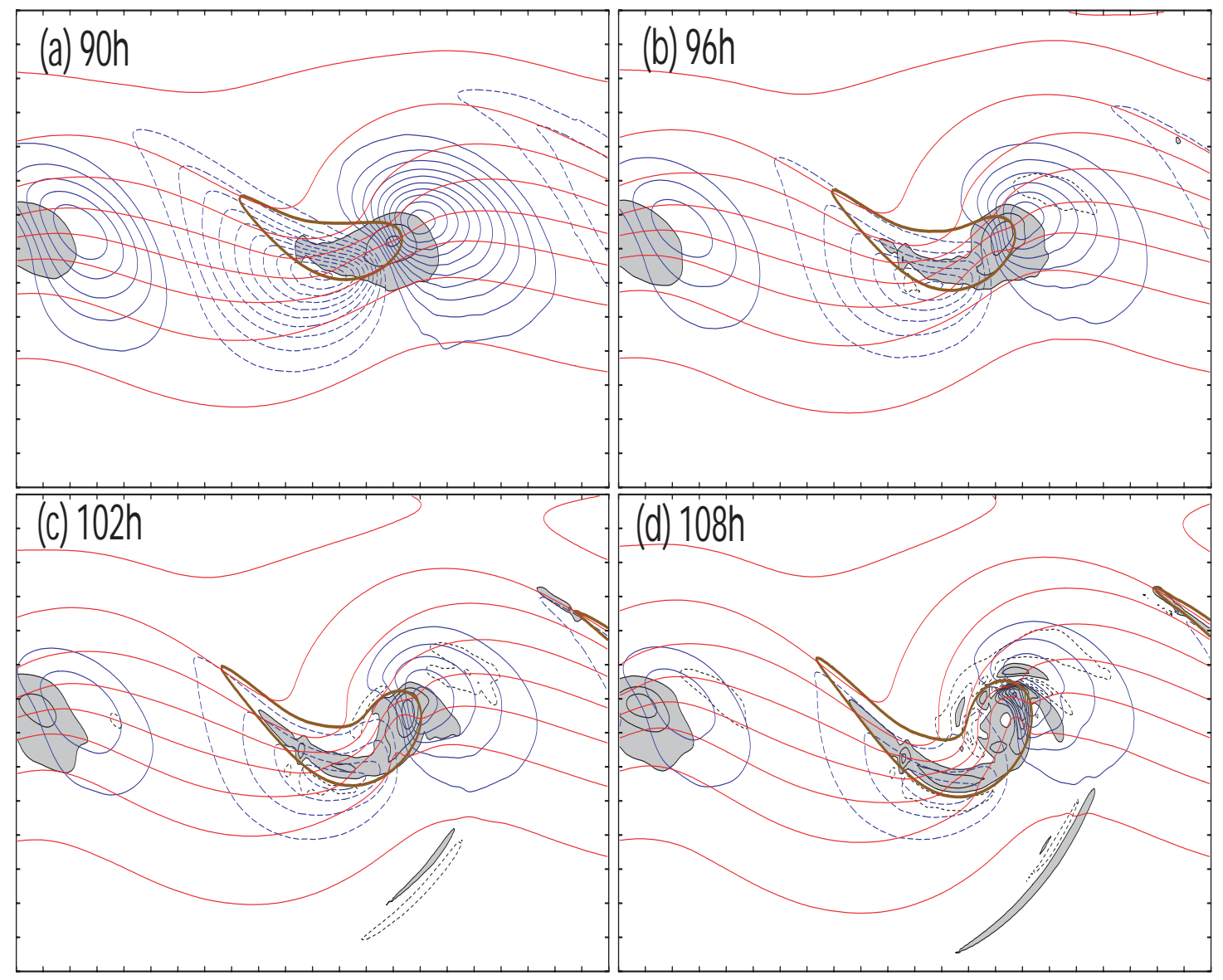

Figure 7: CNTL simulated imbalanced flow at $6 \mathrm{~km}$ at $90 \mathrm{~h}(\mathrm{a}), 96 \mathrm{~h}(\mathrm{~b}), 102 \mathrm{~h}(\mathrm{c})$ and $108 \mathrm{~h}(\mathrm{~d})$. Imbalance $\triangle N B E$ (shaded, solid, positive; dashed, negative; ci $=1 \times 10^{-9} \mathrm{~s}^{-2}$ ), $\theta$ (red solid lines, ci $=5 \mathrm{~K}$ ), vertical velocity(blue thin lines, solid, positive; dashed, negative; $\mathrm{ci}=0.5 \mathrm{~cm} / \mathrm{s}$ in (a), $\mathrm{ci}=1.0 \mathrm{~cm} / \mathrm{s}$ in (b), $\mathrm{ci}=1.5 \mathrm{~cm} / \mathrm{s}$ in $(\mathrm{c}), \mathrm{ci}=2 \mathrm{~cm} / \mathrm{s}$ in (d)); and 1.5PVU (brown thick lines). The distance between tick marks is $300 \mathrm{~km}$. 


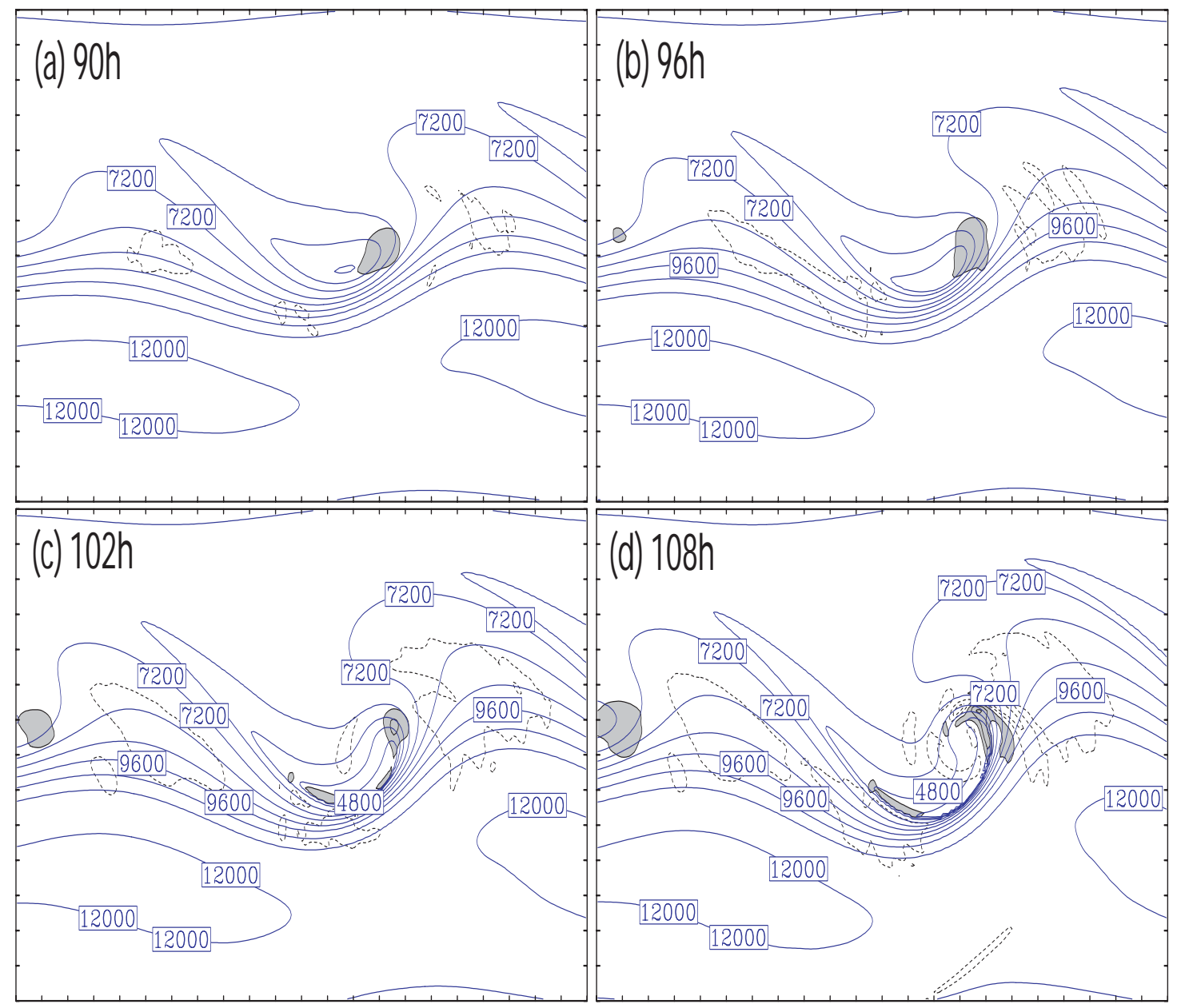

Figure 8: Imbalance on tropopause denoted by 1.5PVU plane. $\triangle N B E$ (shaded, solid, positive; dashed, negative; $\mathrm{ci}=1 \times 10^{-9} \mathrm{~s}^{-2}$ ) and geopotential (blue solid lines, $\mathrm{ci}=800 \mathrm{~m}$ ). The distance between tick marks is $300 \mathrm{~km}$. 
Low frequency inertial GWs during upper level frontogenesis have been discussed by Reeder and Griffiths (1996) and Griffiths and Reeder (1996). They concluded that in their two dimensional model unsteady cross-frontal circulations trigger stratospheric GWs. However, two dimensional cross-frontal circulations can hardly be defined in 3D models especially for a strongly curved jet. In CNTL secondary transverse circulations associated with jet-front actually possess plentiful 3D structures. Therefore it is suggested that GWs are generated due to nonlinear balance adjustment in 3D primitive equations models as an extension to $2 \mathrm{D}$ balance adjustment during frontogenesis.

From a Lagrangian perspective, the pattern of $\triangle N B E$ can also be understood by examining divergence tendency following an air parcel from the troposphere. As a jet streak approaches an upper level ridge, air parcels experience a significant imbalanced period characterized by dramatic increase in divergence. A full divergence equation in pressure coordinate is given by,

$$
\frac{d D}{d t}=-D^{2}-\nabla \omega \cdot \frac{\partial \vec{V}}{\partial P}+2 J(u, v)+f \cdot \zeta-\nabla^{2} \Phi
$$

Each of the last 3 terms, $\triangle N B E$, is much larger than all the other terms in (8). As discussed in RSS94, the warm air parcels ride down isentropes from the stratosphere forming the ridge of isotherms at $6 \mathrm{~km}$. Imbalance among the last three terms forces a large increase in divergence as low PV air parcels ride up from the troposphere and move through the jet exit regions. (Recall that the region of intense gradient of vertical motions at $96 \mathrm{~h}$ coincides with imbalance regions; the same holds for divergence.) Obviously, residence time of parcels in regions of maximum divergence tendency $(\triangle N B E)$ depends on wind speed. 
In a barostrophic or baroclinic atmosphere, nonlinear balance would be a more accurate measure of balance state rather than geostrophy or quasi-geostrophy (Z04 and refs.). To further quantify the link between imbalance (nonlinear balance residue) and gravity waves through balance adjustment, Plougonven and Zhang (2005, personal communications) have recently performed a multiscale analysis of a simplified primitive equation set in a f-plane, adiabatic, inviscid fluid with Boussinesq approximation:

$$
\left\{\begin{array}{c}
\frac{D u}{D t}-f v+\frac{1}{\rho} \frac{\partial P}{\partial x}=0 \\
\frac{D v}{D t}+f u+\frac{1}{\rho} \frac{\partial P}{\partial y}=0 \\
\frac{D w}{D t}+\frac{1}{\rho} \frac{\partial P}{\partial z}+g=0 \\
\frac{\partial u}{\partial x}+\frac{\partial v}{\partial y}+\frac{\partial w}{\partial z}=0 \\
\frac{D \theta}{D t}=0
\end{array}\right.
$$

with

$$
\frac{D}{D t}=\frac{\partial}{\partial t}+\vec{V} \cdot \nabla+w \frac{\partial}{\partial z}
$$

The scaling involves assumptions on separation of two types of motions: a largescale slow-varying component of the flow $\overline{U_{B}}$ which is close to geostrophy and free of GWs, and a mesoscale flow component of the flow U' that describes GWs with a characteristic of a faster timescale of $1 / \mathrm{f}$ or smaller and spatial scales an order of magnitude smaller than that of the larger-scale baroclinic waves. Under this assumption and further neglect high-order nonlinear terms, the simplified primitive equations can be rewritten as: 


$$
\left\{\begin{array}{c}
\frac{\delta_{B} D^{\prime}}{\delta t}-f \cdot \zeta^{\prime}+\nabla^{2} \Phi^{\prime}=\Delta N B E \\
\frac{\delta_{B} \zeta^{\prime}}{\delta t}-f \cdot D^{\prime}=0 \\
D^{\prime}+\frac{\partial w^{\prime}}{\partial z}=0 \\
\frac{\delta_{B} \theta^{\prime}}{\delta t}+\frac{N^{2} \Theta}{g} w^{\prime}=0 \\
\frac{\delta_{B} w^{\prime}}{\delta t}-g \frac{\theta^{\prime}}{\Theta}+\frac{\partial \Phi}{\partial z}=0
\end{array}\right.
$$

where $\frac{\delta}{\delta t}=\frac{\partial}{\partial t}+\overline{U_{B}} \cdot \nabla$, which is the partial derivative following the large-scale flow $\overline{U_{B}}$.

Under the assumption that the gravity wave motions are in a much smaller, faster scale than the large-scale flow, the total derivates in (10) becomes a linear operator. Then a simple gravity wave equation forced by the derivatives of the imbalance residual can be derived from (10):

$$
\left(\frac{\delta_{B}^{2} \nabla^{2}}{\delta t^{2}}+N^{2} \nabla^{2}+\frac{\delta_{B}^{2}}{\delta t^{2}} \frac{\partial^{2}}{\partial z^{2}}+f^{2} \frac{\partial^{2}}{\partial z^{2}}\right) w^{\prime} \approx \frac{\partial}{\partial z} \frac{\delta_{B}}{\delta t}(-\Delta N B E)
$$

Details of this analytic derivation and the underlying assumptions will be reported by Plougonven and Zhang after its completion. This analytical approach identifies a direct link between the forcing by large scale imbalance and the generation of smaller scale gravity waves. Under this analysis, the gravity wave characteristics (frequency and wavelength) must be determined or modulated by the imbalance structure and variation. The imbalance by itself is a product of the large-scale baroclinic waves. This preliminary analytical approach thus partially motivates the following experiments to explore the direct relationships between the larger-scale baroclinic waves (and the attendant imbalance) and the mesoscale gravity waves generated during different life cycles. 


\section{SENSITIVITY OF GRAVITY WAVES TO THE BAROCLINICITY}

\subsection{Overview of simulated baroclinic waves}

Besides the control experiment discussed in Z04 and the previous section, 4 additional life cycles of baroclinic waves initialized with different background shear and static stability have been simulated. Control of the background baroclinicity is achieved through altering the geometry or steepness of the initial tropopause (eq (4); refer to as "S") and tropospheric PV ( $P V_{T}$ in eq(3); equivalent to change in tropospheric static stability, refer to as "N") before the 2-D PV inversion was performed (section 2).

We will examine GW sensitivity to the background baroclinicty in comparison to the control experiment. If either of the two determining parameters (i.e., $\mathrm{S}$ and $\mathrm{N}$ ) in the initial conditions of CNTL is modified, the subsequent growth rate of the BWs may differ significantly from that of CNTL. More specifically, experiment "BigS" ("LowS") employs a steeper (flatter) initial tropopause that results in a stronger (weaker) jet streak and thus stronger (weaker) vertical wind shear (Fig. 2b-c). Experiment "LowN" reduced the initial tropospheric PV and static stability in CNTL by half (Fig. 2d). Both the slope of tropopause and tropospheric PV are changed from CNTL in experiment "LowNlowS" with a lower tropospheric stability and a flatterer initial tropopause (Fig. 2e) so that close growth rate of baroclinic waves is simulated.

Despite expected differences in phase, scale and intensity, typical life cycles of dry baroclinic waves similar to that observed in CNTL have been simulated in all the aforementioned sensitivity experiments (not shown). Moreover, mesoscale gravity waves with different wave characteristics have been simulated in the exit region of the uppertropospheric jet streaks for all these experiments (detailed below). 
The evolution from balanced initial state to strong imbalance in the vicinity of upper-level jet-front (and tropopause) preceding the mesoscale gravity waves is also qualitatively similar in all the sensitivity experiments. Figure 9 shows the surface features when imbalance become well developed in all cases, approximately 9-12 hours before mature GWs appear in the vertical motion and temperature fields at upper levels (refer to table 2 for display time for each case). At this time, the surface cyclones in the five cases have all been well developed with a minimum MSLP of 940-965 hPa. Warm air is beginning to be wrapped in to form warm cores by cyclonic circulations. Surface occluded fronts form "T bone" structures. The horizontal wavelength of the BWs for all the experiments is similar to CNTL $(\sim 3900 \mathrm{~km})$ and is summarized in Table 2.

The horizontal wind speeds at $300 \mathrm{hPa}$, which is the level of maximum jet strength in the initial conditions, are shown in Fig. 10 at the same times as for Fig. 9, which are $\sim 10$ hours earlier than the appearance of mature GWs (detailed below). Upper level features display great variability in the strength and shapes of the jets. In the presence of a stronger jet, a flat flow with less curvature appears in BigS, LowN (Fig. 10 $\mathrm{b}$ and d). In Fig. $10 \mathrm{c}$ and e, winds downstream of the trough turn northward. Also at the same times, divergence fields at $13 \mathrm{~km}$ (Fig. 11) in developing BWs show divergence downstream of the trough with weaker signals of gravity waves beginning to emerge in the exit region of the jet streaks. These weaker wave signals will evolve into coherent mature gravity waves 9 to $12 \mathrm{~h}$ later at the 13-km divergence field (Fig. 12).

As in Z04 the initial tropopause-level PV anomaly develops into a primary cyclone, accompanied by secondary cyclogenesis both upstream and downstream. Evolution of the minimum sea level pressure (SLP) of the major low pressure center 
Table 2: Summary of characteristics for baroclinic waves for five experiments. Column 1 is the list of experiments. Column 2 lists the times of maximum imbalance when surface and $300 \mathrm{hPa}$ are shown in Figs. 10-11. Columns 3 and 4 are the maximum wind speeds at 300hp and minimum sea level pressure (MSLP) at these times. Column 5 is the time mature gravity waves are shown in Fig. 16. Column 6 is MSLP at the times listed in Column 5. Column 7 is the wavelength of the baroclinic wave. Columns 8 and 9 list the averaged quasi-linear growth rates and the period during which the averaged qiasi-linear growth rates are estimated.

\begin{tabular}{|c|c|c|c|c|c|c|c|c|}
\hline Expr & $\begin{array}{c}\text { Maximum } \\
\text { imbalance } \\
\text { time } \\
\text { (hour) }\end{array}$ & $\begin{array}{c}\text { Jet } \\
\text { strength } \\
(\mathrm{m} / \mathrm{s})\end{array}$ & $\begin{array}{c}\text { MSLP } \\
(\mathrm{hPa})\end{array}$ & $\begin{array}{c}\text { Mature } \\
\text { GWs } \\
\text { time } \\
\text { (hour) }\end{array}$ & $\begin{array}{c}\text { MSLP } \\
(\mathrm{hPa})\end{array}$ & $\begin{array}{c}\text { Wavelength } \\
\text { Of BWs } \\
(\mathrm{km})\end{array}$ & $\begin{array}{c}\text { Averaged } \\
\text { Growth } \\
\text { rate } \\
\text { (time) }\end{array}$ & $\begin{array}{c}\text { Period } \\
\text { for } \\
\text { average } \\
\text { (hour) }\end{array}$ \\
\hline CNTL & 102 & 45.9 & 948 & 114 & 934 & 3900 & 0.35 & $45-90$ \\
\hline BigS & 78 & 52.4 & 948 & 90 & 927 & 3900 & 0.4 & $40-70$ \\
\hline LowS & 132 & 39.4 & 953 & 144 & 944 & 3600 & 0.28 & $60-120$ \\
\hline LowN & 39 & 56.2 & 967 & 48 & 948 & 3600 & 0.58 & $36-48$ \\
\hline LowNlowS & 99 & 40.3 & 951 & 111 & 940 & 3900 & 0.32 & $45-90$ \\
\hline
\end{tabular}



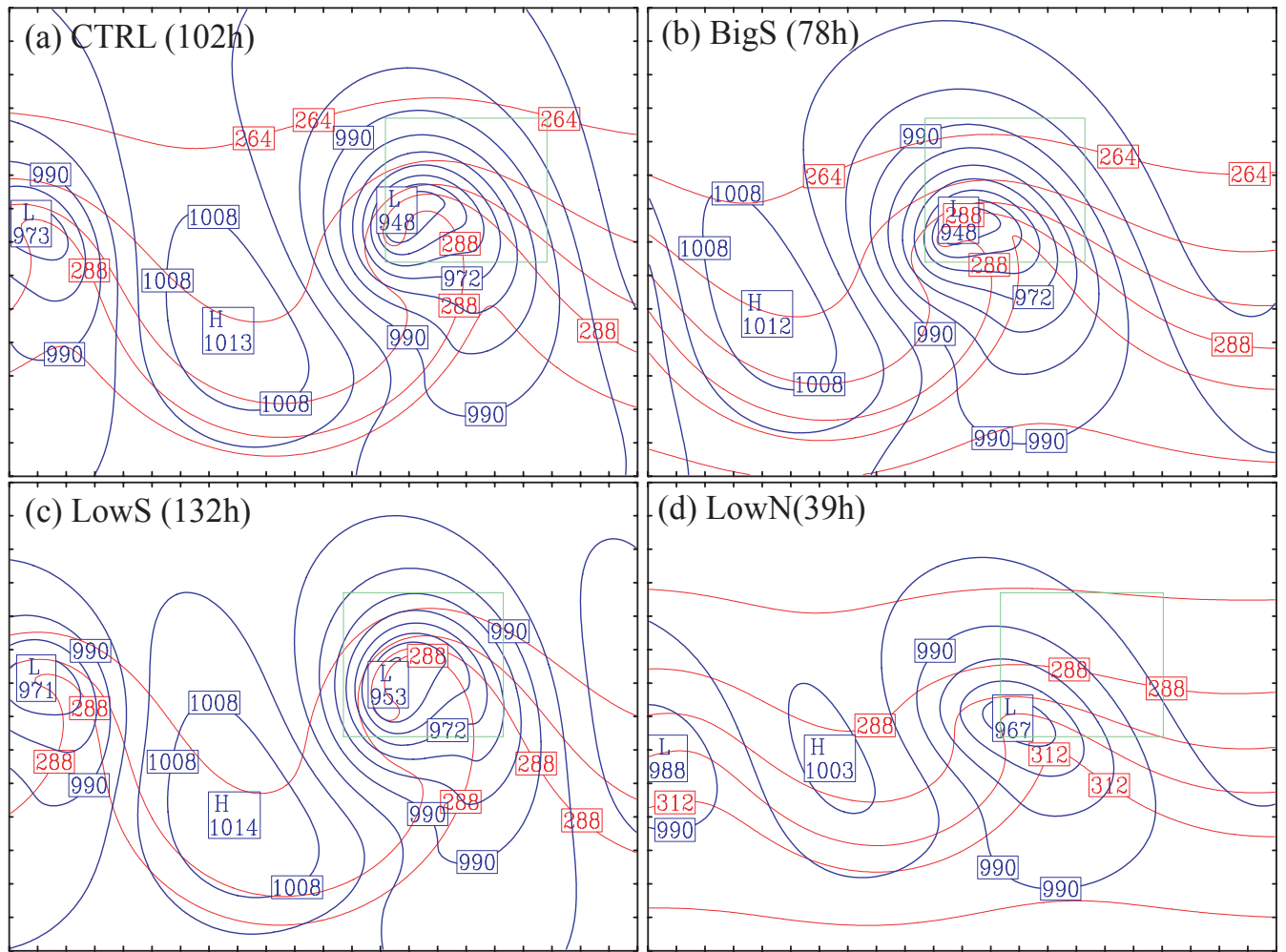

(d) $\operatorname{LowN}(39 \mathrm{~h})$
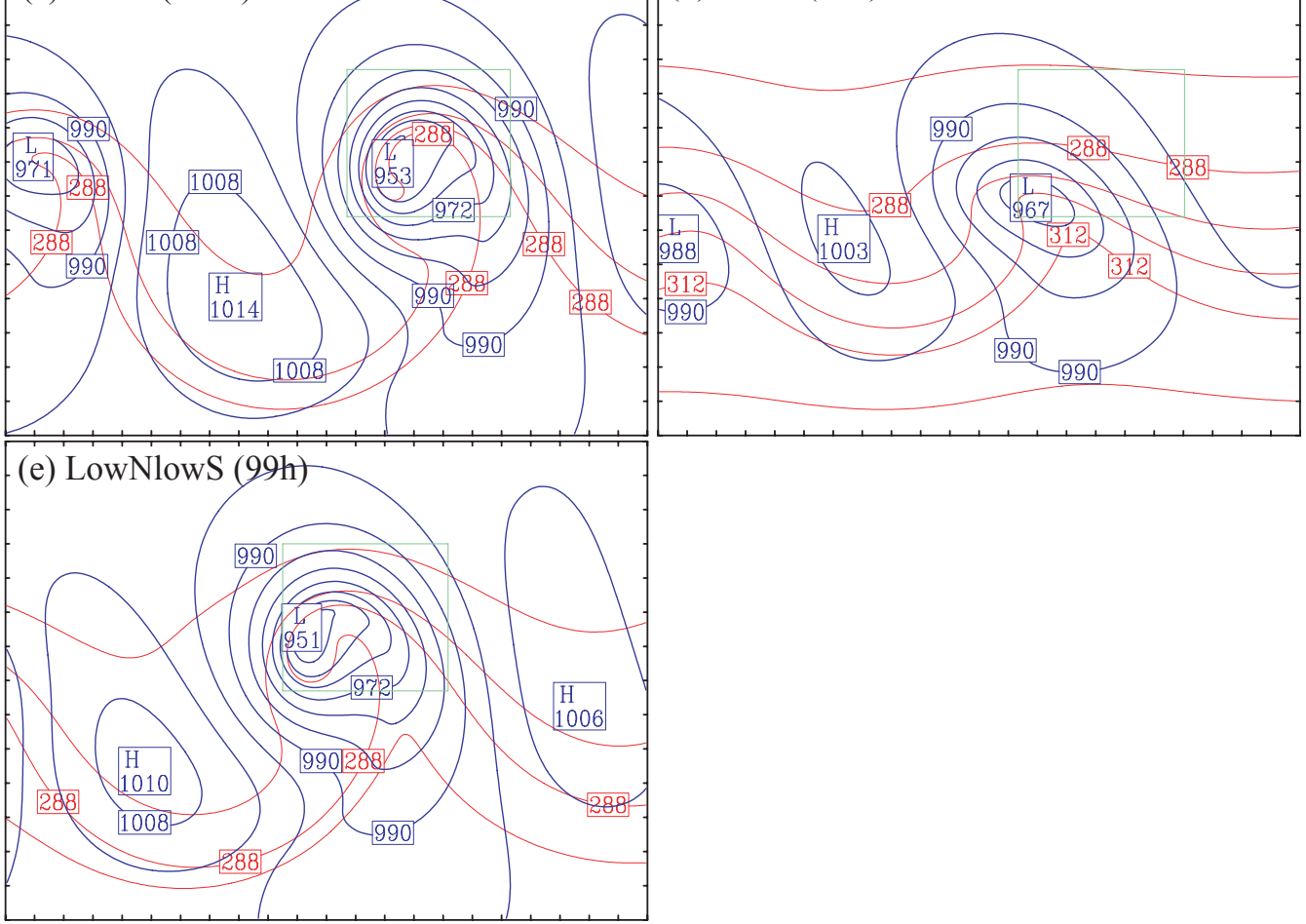

Figure 9: Surface features for all the five cases. Potential temperature $\theta$ (red thin lines, $\mathrm{ci}=8 \mathrm{~K}$ ) and sea level pressure (Blue thin lines, $\mathrm{ci}=6 \mathrm{hPa}$ ). Green boxes denote the area in Figs. 15-18. The distance between tick marks is $300 \mathrm{~km}$. 

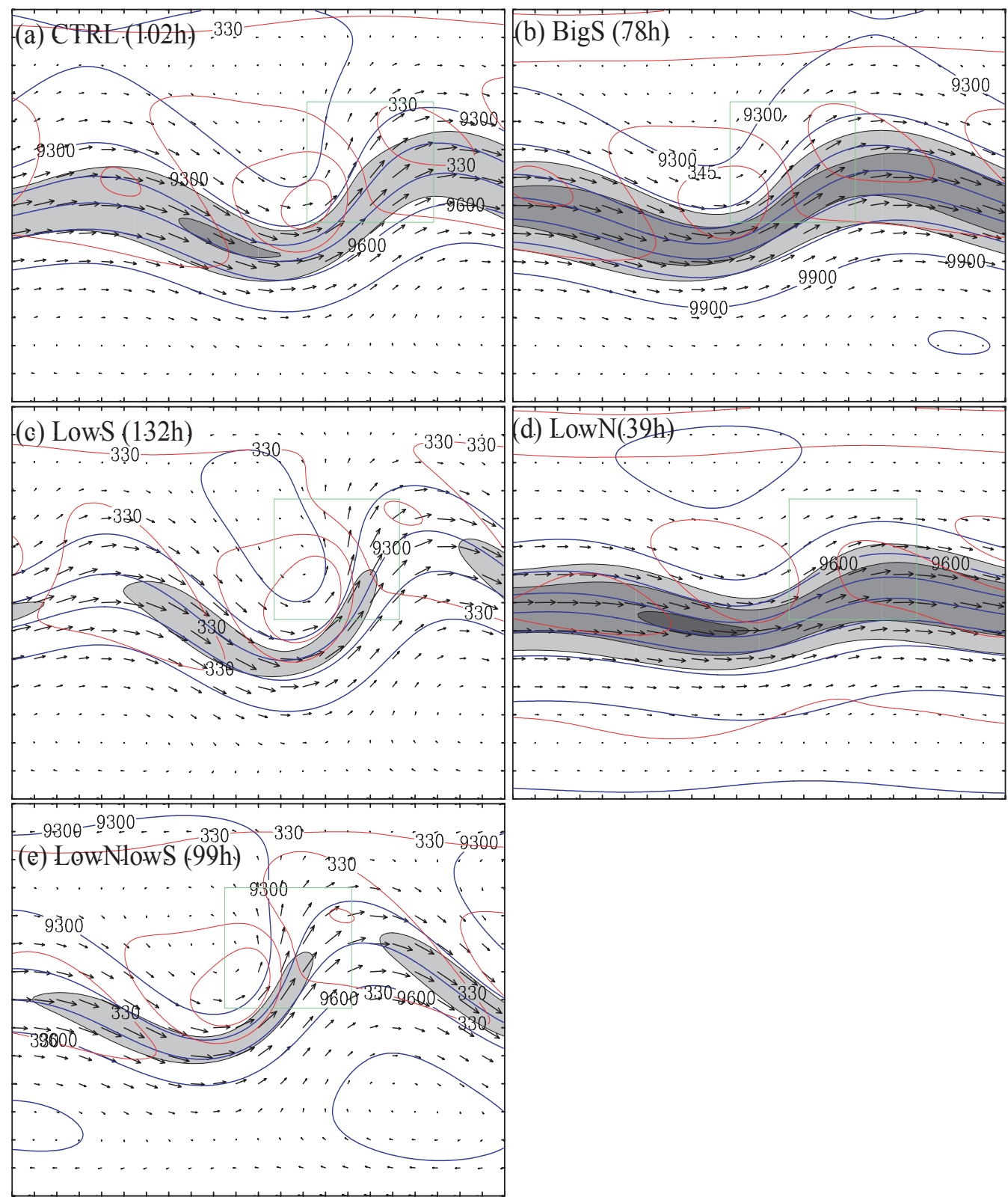

Figure 10: Upper level jet. Isotachs (values greater than $35 / \mathrm{ms}$ light shaded, dark shaded regions are values greater than $55 \mathrm{~m} / \mathrm{s}$ for a-h, $65 / \mathrm{ms}$ for $\mathrm{i}, \mathrm{j}$ ), wind vector, geopotential (thick lines, ci $=100 \mathrm{~m}$ ) and $\theta$ (thin lines, $\mathrm{ci}=5 \mathrm{k}$ ) on $300 \mathrm{hPa}$. The distance between tick marks is $300 \mathrm{~km}$. 

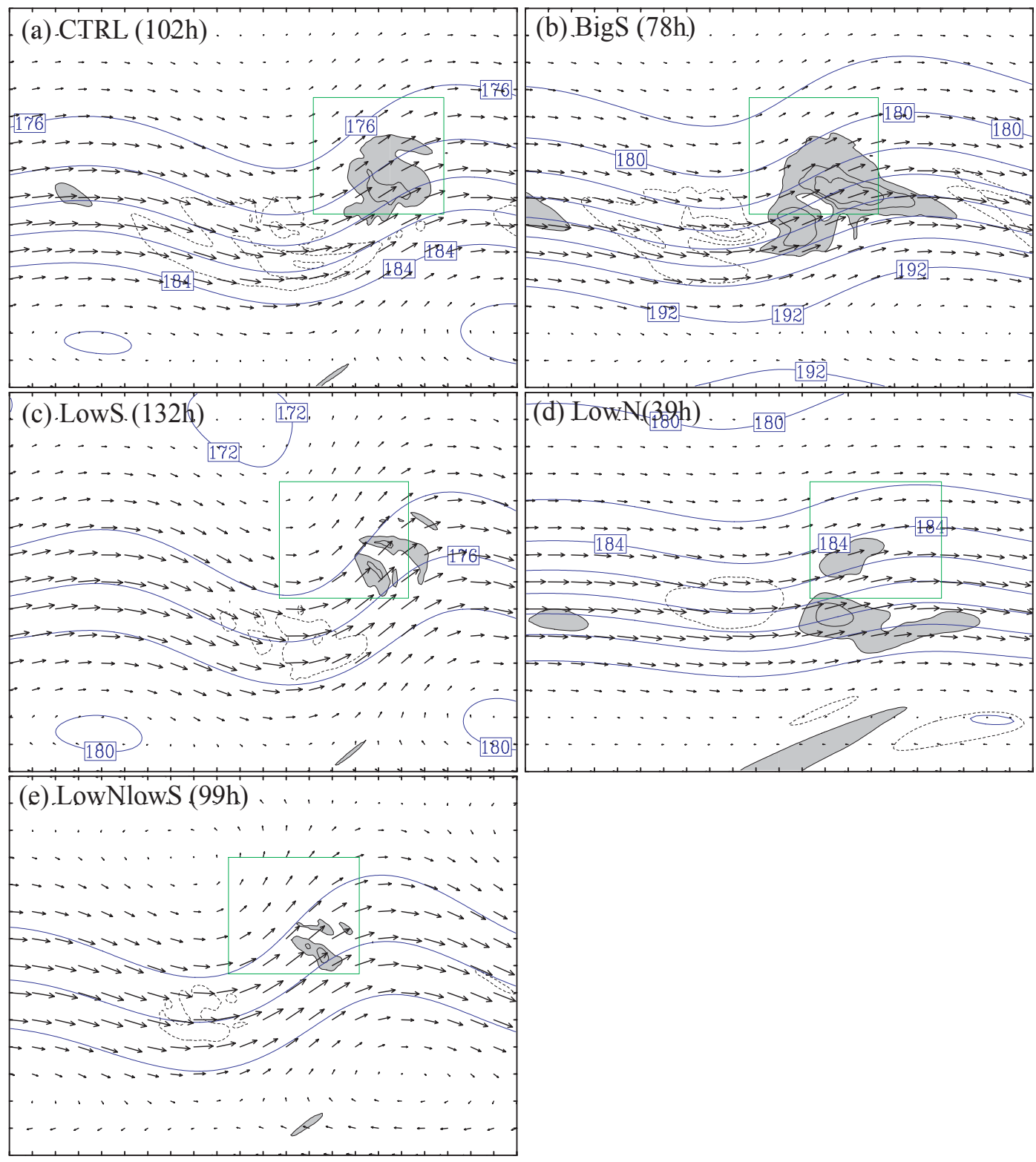

Figure 11: Divergence pattern 9-12 hours before gravity waves are plotted in Fig. 15. Divergence (thin line; solid and shaded, positive; dashed, negative; ci $=2 \times 10^{-6} \mathrm{~s}^{-1}$ ), pressure (thick line, $\mathrm{ci}=2 \mathrm{hPa}$ ) and wind vectors. The distance between tick marks is 300 $\mathrm{km}$. 

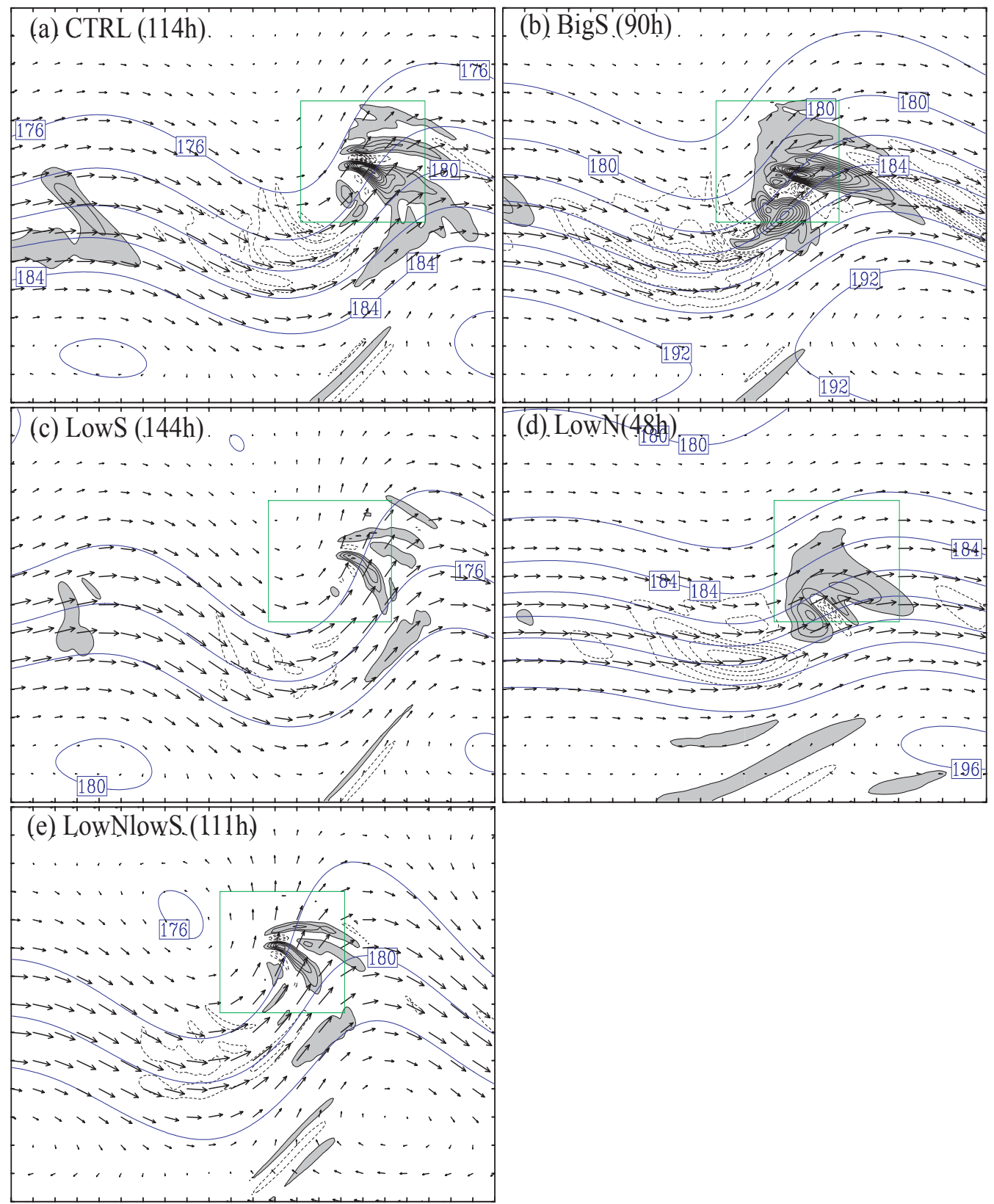

Figure 12: Divergence pattern at times gravity waves are displayed in Fig. 15. Divergence (thin line; solid and shaded, positive; dashed, negative; ci $=2 \times 10^{-6} \mathrm{~s}^{-1}$ ), pressure (thick line, $\mathrm{ci}=2 \mathrm{hPa}$ ) and wind vectors. The distance between tick marks is 300 $\mathrm{km}$. 
estimated in Domain 2 for all cases are shown in Fig. 13a, which gives a preliminary picture of the growing baroclinic in all experiments. By design, through changing either the slope of the tropopause or the tropospheric PV in the initial conditions (BigS, LowS and LowN), the growth rate the resulting baroclinic waves (Table 1) differs significantly from that of the CNTL case. Experiment LowNlowS modified both factors simultaneously to achieve a slightly small growth rate to CNTL (Fig. 13a).

To track the intensity and growth of the BWs from the model initial time, we also compute the minimum pressure perturbation of the primary surface cyclone in Domain 1 by subtracting zonal mean SLP from the SLP field. Time series of the maximum pressure perturbation (in absolute value) of the primary cyclones are shown in Fig. 13b for all the five cases. Then, a global exponential growth rate of BWs can be defined by

$$
\sigma=\frac{\log \left(\frac{\delta P_{t+1}}{\delta P_{t}}\right)}{\Delta t}
$$

where $\mathrm{P}_{t}$ and $\mathrm{P}_{\mathrm{t}+1}$ are the maximum perturbation surface pressure for cyclones at the model output times of $t$ and $t+1$ every $6 \mathrm{~h}$. This definition differs from the growth rate used by Badger and Hoskins (2001) in that we use maximum surface pressure perturbation rather than kinetic energy. Fig. 14 shows the evolution of the growth rates of BWs including the CNTL case, which also display similar growth pattern for all five experiments. For example, in the CNTL case, an initial rapid growth period, similar to that termed as non-modal growth (Farrell 1982), appears in the first 12 hours, which is followed by a period of $\sim 70-90 \mathrm{~h}$ during which the growth rate asymptotes to a constant value. This mechanism of quasi-linear growth is close to the so-called modal growth mechanism although in this case the boundary conditions in the zonal directions are not 

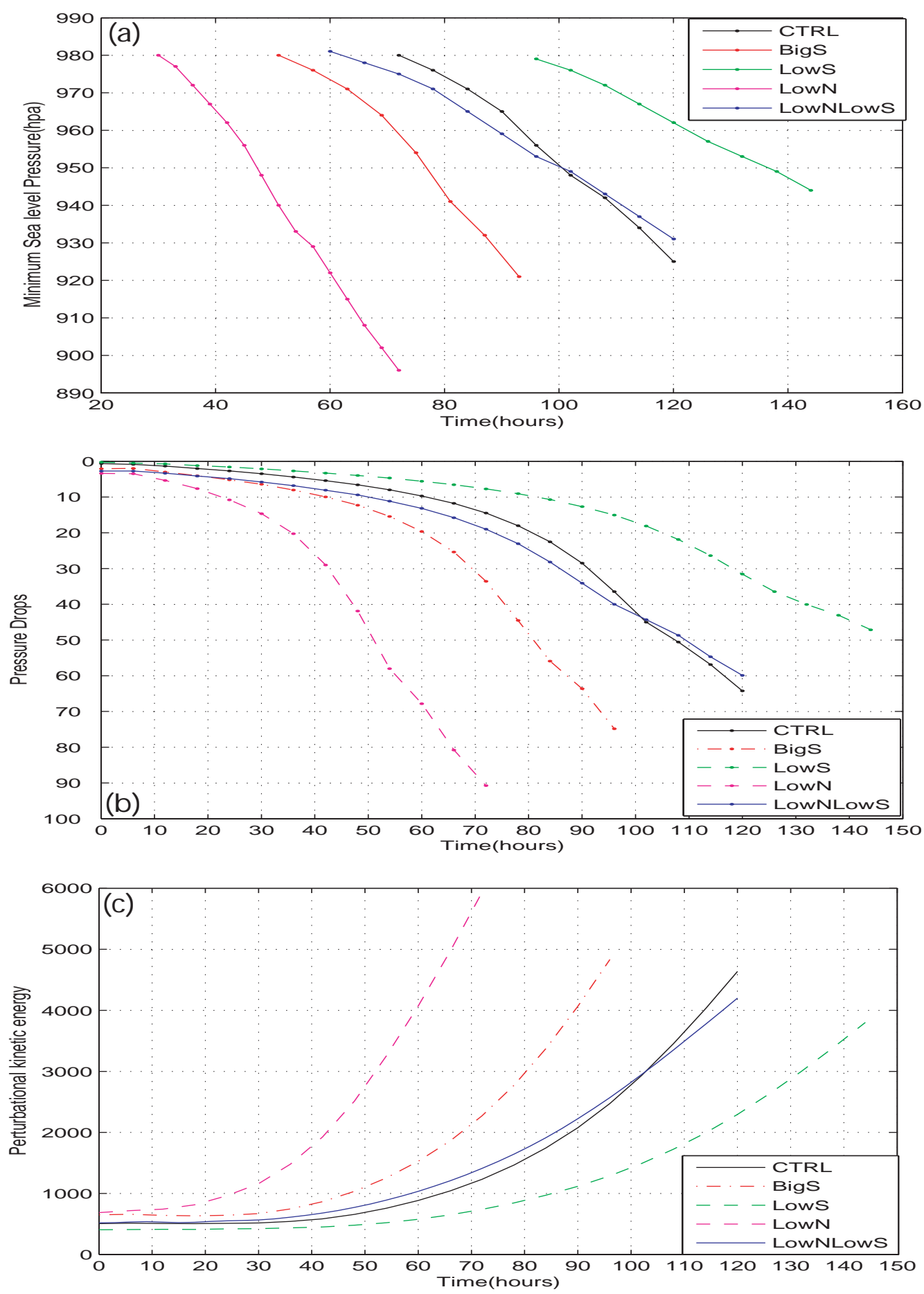

Figure 13: (a) Time series of MSLP (minimum sea level pressure) in Domain 2 for 5 experiments. (b) Minimum perturbation pressure of the cyclones in Domain1 (hPa). (c) Overall perturbation kinetic energy from surface to upper levels $(15 \mathrm{~km})$. 

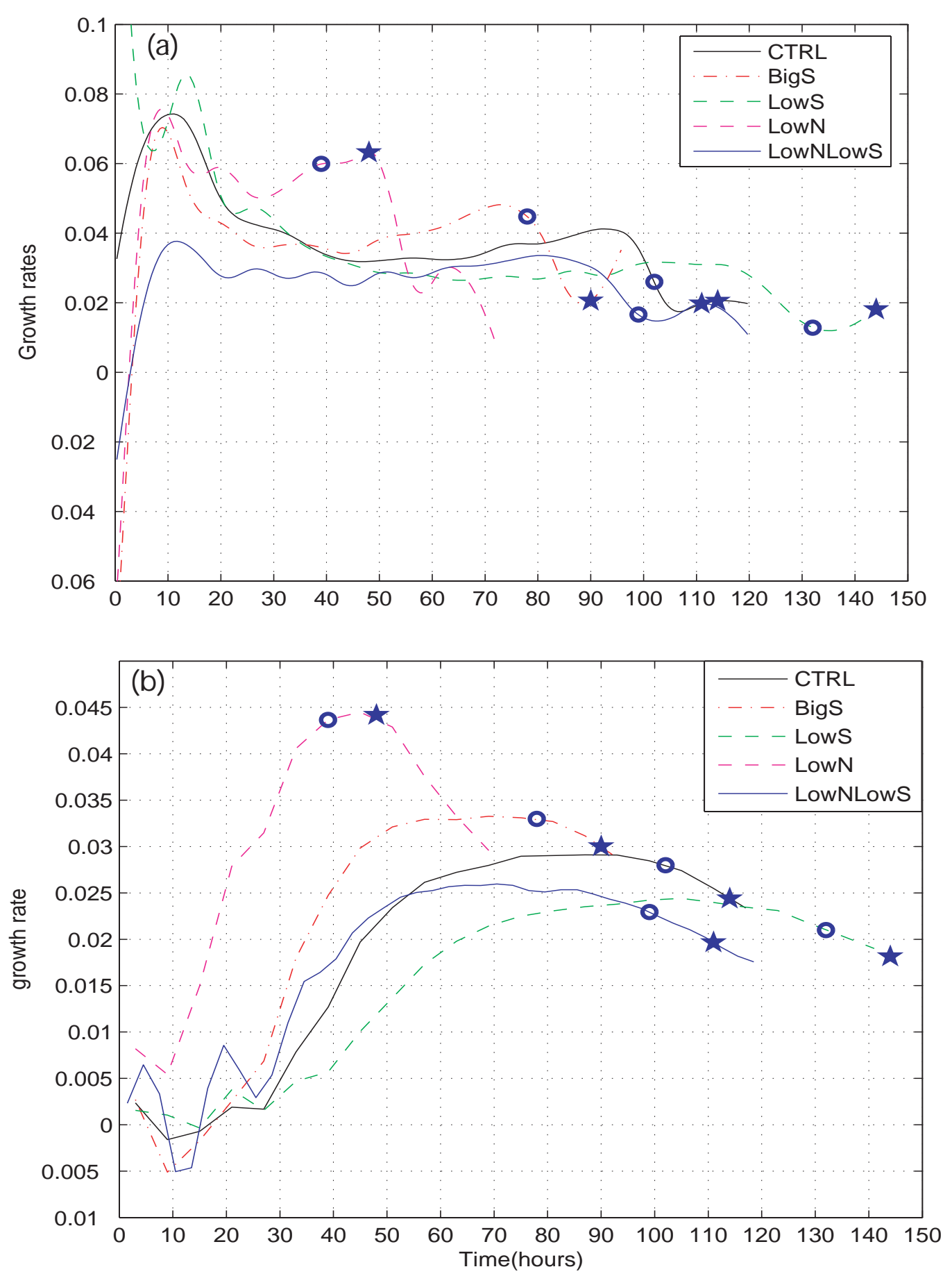

Figure 14: Growth rates in terms of (a) perturbation pressure and (b) perturbation kinetic energy for five experiments. Stars indicate the timing for each case in Fig. 20. Big circles indicate the timing GWs are shown in Fig. 15. 
periodic. At around 90 hour, the growth rate drops significantly signaling the approaching of baroclinic saturation. Figure 14 further shows the quasi-linear growth rates of LowN and BigS differ from that of the CNTL, but the growth rates of LowS and LowSlowN are very similar to CNTL. To be noted that, the growth rate defined in terms of pressure could be different that in terms of kinetic energy for growing BWs as pointed out by Rotunno and Bao (1996). Hakim (2000) demonstrated that growth rates defined in four different norms shows considerable difference.

Perturbation wind components u', v' and w' can also be computed in a similar way to the calculation of perturbation SLP. The perturbation kinetic energy of BWs is the sum of kinetic energies $\left(K^{\prime}=(1 / 2)\left[\left(u^{\prime}\right)^{2}+\left(v^{\prime}\right)^{2}+\left(w^{\prime}\right)^{2}\right]\right)$ from the surface to upper levels. Fig 13c shows perturbation kinetic energy increases nearly exponentially for these five cases. The growth rates of BWs are thus calculated from the perturbation kinetic energy in the same way as from perturbation pressure (Fig. 14b). Not surprisingly, the growth rates in terms of perturbation kinetic energy differ from grow rates in terms of perturbation SLP.

\subsection{Overview of gravity waves in the five cases}

Similar to CNTL, mesoscale gravity waves have been generated during all the different life cycles of the baroclinic waves. These waves first begin to be visible in the strong divergent region in the exit region of the upper-tropospheric jet streaks and downstream of the upper-level trough (Fig. 11) that evolve into several distinct wave crests and troughs 9-12 hours later (Fig. 12). Figure 15 shows a zoomed-in display (green boxes in Fig. 9-12) of the mature gravity waves in the vertical velocity and potential 

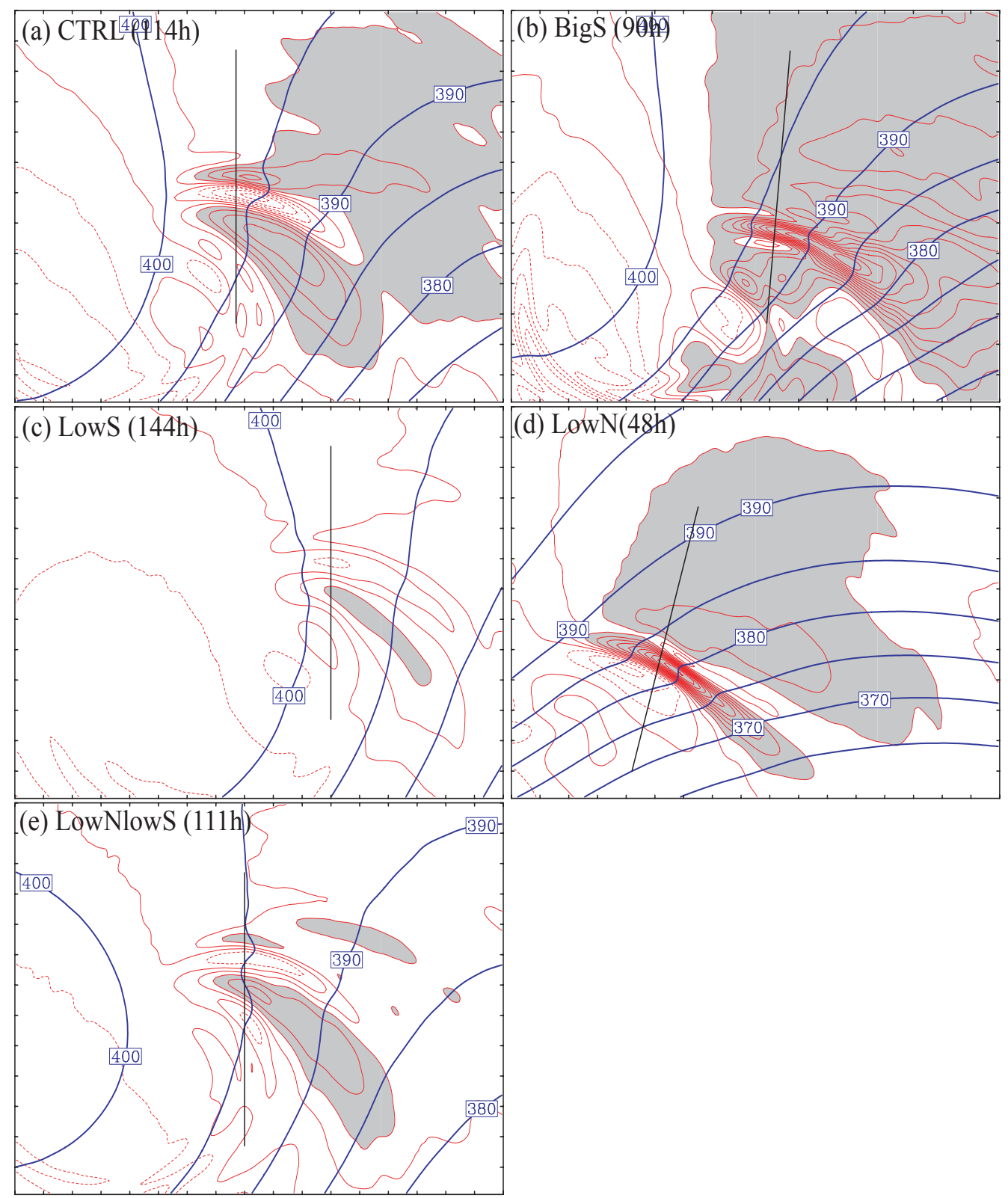

Figure 15: Vertical velocities (solid, positive; dashed, negative; $\mathrm{ci}=2 \times 10^{-3} \mathrm{~m} / \mathrm{s}$; values $>5 \times 10^{-3} \mathrm{~m} / \mathrm{s}$ shaded) and $\theta$ (blue thick line, ci $=5 \mathrm{~K}$ ) on $13 \mathrm{~km}$ in Domain 3 . Timing of plots is the same as in Fig. 10. The area is indicated by green box in Figs. 9-12. The distance between tick marks is $100 \mathrm{~km}$. 
temperature fields at $13 \mathrm{~km}$ for all cases with display of each experiment listed in Table 2. The corresponding vertical structures of the gravity waves at the same times are displayed in Fig. 16. The times are selected based on the presence of two or more crests and troughs of relatively large amplitude prior or the appearance of waves with other wavelengths. Since the GWs are unavoidably mixed with strong larger-scale ascent in this region, we perform a nine-point smoothing repeatedly (21 times) and subtract the smoothed fields from the unsmoothed fields to derive clear gravity wave signals at the mesoscale (Figs. 17-18). The response function for the filter using nine-point weighted smoother (P397, Haltiner and Williams, 1980) is

$$
R(k)=1-\left(\left(1-\sin ^{2} k \Delta x / 2\right)\left(1-\sin ^{2} k \Delta y / 2\right)\right)^{21}
$$

where $\mathrm{k}$ is the wave number, $\Delta \mathrm{x}, \Delta \mathrm{y}$ are the horizontal grid spacing $(10 \mathrm{~km})$. Figure 19 shows the response function (15) versus horizontal wavelengths. Plane waves with horizontal wavelength $150 \mathrm{~km}$ have reduced magnitude around 0.8 times of original signals. Magnitude of the waves with horizontal wavelength $200 \mathrm{~km}$ is reduced to about 0.65 of their original magnitude. Common mesoscale gravity waves have wavelengths from $50 \mathrm{~km}$ to $500 \mathrm{~km}$. This filter can significantly damp out long waves (e.g. for waves having wavelengths great than $200 \mathrm{~km}$ ). Since we are focusing the gravity waves with wavelengths less than $200 \mathrm{~km}$ (e.g. GWs in CNTL have a horizontal wavelength of 150 $\mathrm{km}$ in Z04), the filter can be safely applied to extract the wave signals in this study.

By combining surface and $300 \mathrm{hPa}$ features, we conclude that a universal flow configuration for mesoscale GWs consists of surface fronts, upper level jet-fronts and an upper level trough, a pattern identical to UK87. Resulting perturbation vertical motions 

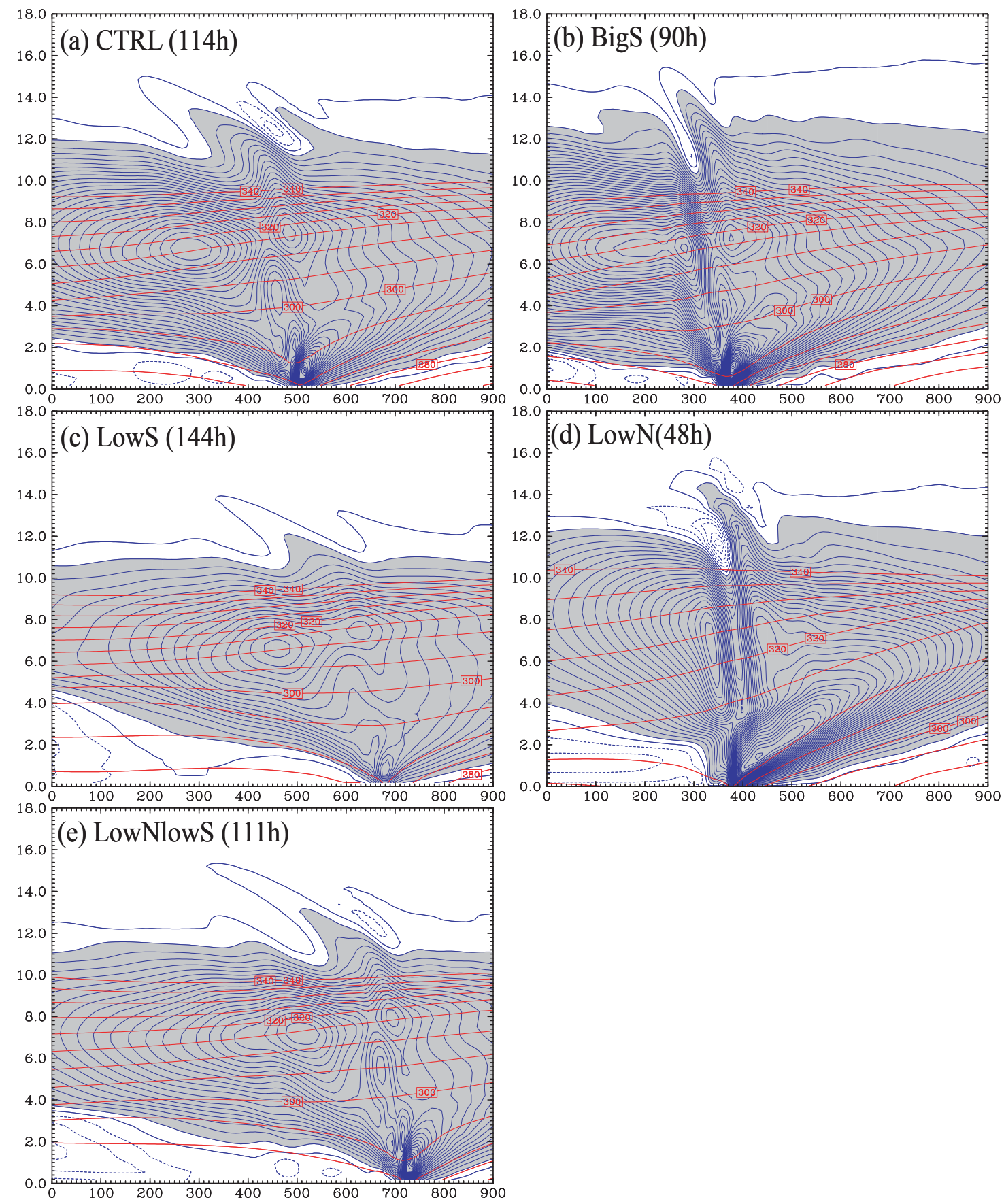

Figure 16: Vertical velocities (solid, positive; dashed, negative; $\mathrm{ci}=5 \times 10^{-3} \mathrm{~m} / \mathrm{s}$; values $>5 \times 10^{-3} \mathrm{~m} / \mathrm{s}$ shaded) and $\theta$ (red thick line, ci $=5 \mathrm{~K}$ ) along cross sections indicated in Fig. 15. 

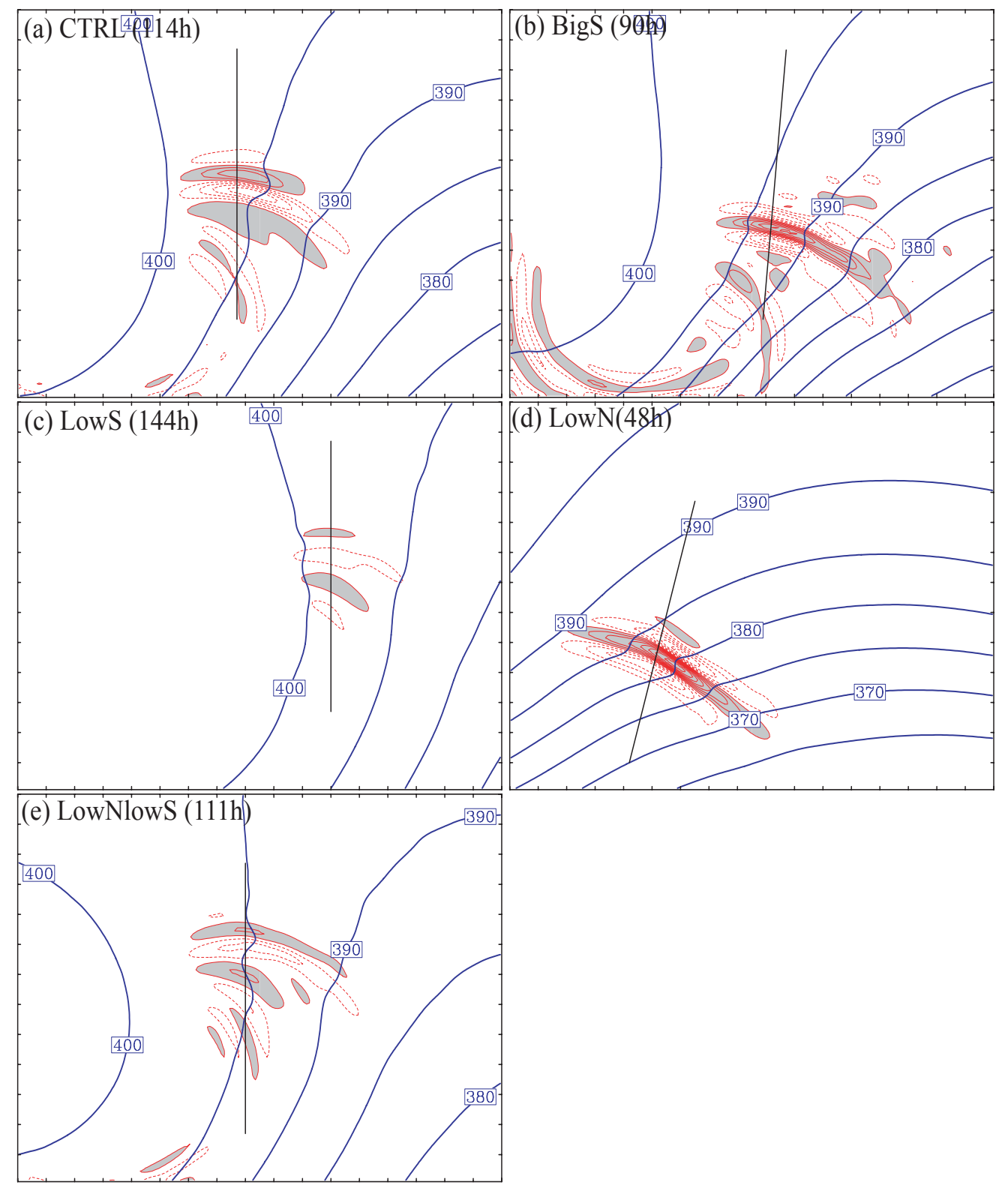

Figure 17: Perturbation vertical velocities $w^{\prime}$ (solid, positive; dashed, negative; ci= $1 \times 10^{-3} \mathrm{~m} / \mathrm{s}$; values $>0.5 \times 10^{-3} \mathrm{~m} / \mathrm{s}$ shaded) and $\theta$ (blue thick line, ci $=5 \mathrm{~K}$ ) on $13 \mathrm{~km}$. The distance between tick marks is $100 \mathrm{~km}$. 

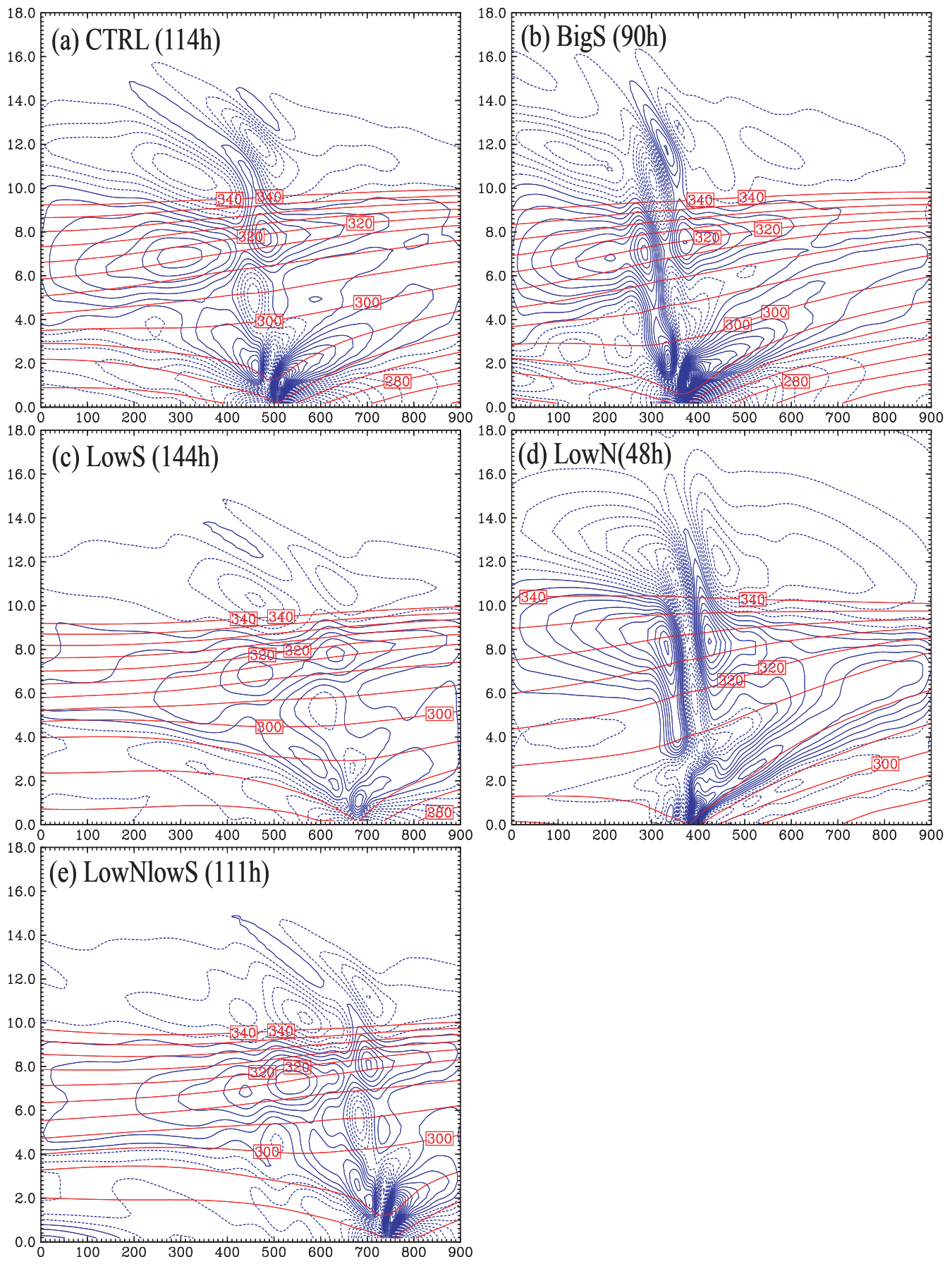

Figure 18: Perturbation vertical velocities $w^{\prime}$ (solid, positive; dashed, negative; ci= $2 \times 10^{-3} \mathrm{~m} / \mathrm{s}$ ) and $\theta$ (thick lines, ci $=5 \mathrm{~K}$ ) along cross sections indicated in Fig. 15 . 


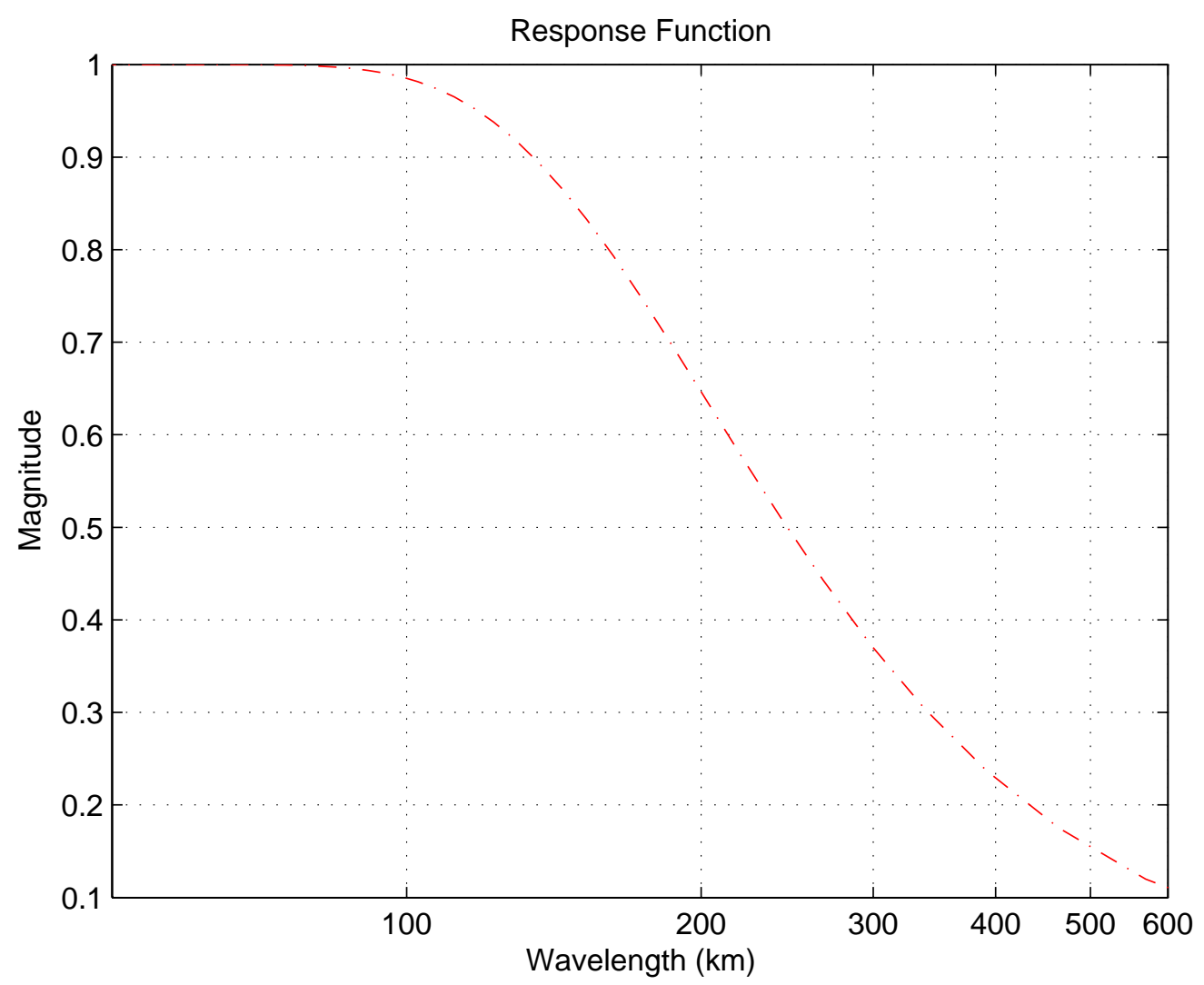

Figure 19: Response function (15) for the filter used in Figures 17, 18 and 21. 
(after filtering) considered as GW signals are shown in Fig. 17. Apparently vertical velocities and potential temperature perturbations are 90 degrees out of phase in Fig. 17 indicative of freely vertical propagating gravity waves. However, there are also other wave signals other than those from the jet streaks present in the simulations. For example, gravity waves in the lower left corner of Fig. $17 \mathrm{~b}$ apparently coming from surface frontogenesis. Waves with distinct horizontal wavelength also appear on the trail of leading GWs. The current study focuses only on those quasi-monochromatic waves apparently generated from the upper-level jet-front system.

Straight lines that cut across the phase front indicate the leading wave signals, which propagate to the upper levels earlier than any other wave signals. Vertical cross sections in Fig. 18 along the straight lines on each panel of Fig. 15 show vertical velocities and potential temperature from south to north (vertical motions have been already filtered horizontally). The filtered vertical motion shows the GW vertical motion mostly uncontaminated by non-wave circulations. We estimate horizontal and vertical wavelengths in the directions perpendicular to the phase front by measuring the distance horizontally and vertically between the maximum and minimum perturbations at or near $13 \mathrm{~km}$. For each case, wavelengths and standard deviations are determined from 10 samples (10 cross sections perpendicular to the wave fronts approximately centered on the lines drawn in Fig. 15 with $10 \mathrm{~km}$ horizontal spacing). These GW wavelengths and the standard variation so measured are listed in Table 3.

Average values of horizontal and vertical wavelengths can be used to derive the intrinsic frequencies assuming linear wave dispersion and hydrostatic approximation.

$$
\omega_{i}^{2}=f^{2}+N^{2} \frac{k^{2}}{m^{2}}=f^{2}+N^{2} \frac{\lambda_{h}{ }^{2}}{\lambda_{z}{ }^{2}}=f^{2}+N^{2} A^{2}
$$


Table 3: Summary of wave characteristics for gravity waves for five experiments. Row 2 is the times when GWs are shown in Fig. 15. Rows 3-6 are horizontal wavelengths with the standard deviation, vertical wavelengths with the standard deviation, intrinsic frequency and phase speed $c_{i}$ derived from dispersion relation. Rows 7-9 are mean wind speeds for GWs, ground relative phase speeds of GWs and mean flow relative phase speed based on columns 7 and 8 . Rows 10 is the growth rate of $\Delta$ NBE calculated from Fig 22.

\begin{tabular}{|c|c|c|c|c|c|}
\hline Expr & CNTL & BigS & LowS & LowN & LowNlowS \\
\hline Mature GWs time (hour) & 114 & 90 & 144 & 48 & 111 \\
\hline$\lambda_{h}(\mathrm{~km})$ & $\begin{array}{c}143.3 \\
( \pm 5.37)\end{array}$ & $\begin{array}{c}96.7 \\
( \pm 1.95)\end{array}$ & $\begin{array}{c}175 \\
( \pm 6.73)\end{array}$ & $\begin{array}{c}79.5 \\
( \pm 4.98)\end{array}$ & $\begin{array}{c}143.2 \\
( \pm 9.2)\end{array}$ \\
\hline$\lambda_{z}(\mathrm{~km})$ & $\begin{array}{c}2.28 \\
( \pm 0.16)\end{array}$ & $\begin{array}{c}3.77 \\
( \pm 0.22)\end{array}$ & $\begin{array}{c}2.2 \\
( \pm 0.0)\end{array}$ & $\begin{array}{c}3.58 \\
( \pm 0.06)\end{array}$ & $\begin{array}{c}2.2 \\
( \pm 0.0)\end{array}$ \\
\hline$\omega\left(10^{-4} \mathrm{~s}^{-1}\right)$ & 3.70 & 8.80 & 3.0 & 10.1 & 3.57 \\
\hline$C_{i}(\mathrm{~m} / \mathrm{s})$ & 8.4 & 13.5 & 8.3 & 12.8 & 8.0 \\
\hline \begin{tabular}{c} 
Mean Wind Speed $(\mathrm{m} / \mathrm{s})$ \\
\hline$c_{g d}(\mathrm{~m} / \mathrm{s})$
\end{tabular} & 3.7 & -0.5 & -0.8 & 7.1 & 0.8 \\
\hline \begin{tabular}{c} 
Observed Phase Speed $(\mathrm{m} / \mathrm{s})$ \\
\hline $\begin{array}{c}\text { Growth rates of } \Delta \mathrm{NBE} \\
\text { maximum }(\text { hour }\end{array}$
\end{tabular} & 8.3 & 13.5 & 8.8 & 13.9 & 8.2 \\
\hline
\end{tabular}

where $\lambda_{h}$ and $\lambda_{z}$ are horizontal and vertical wavelength. A denotes the aspect ratio. Static stability in stratosphere is universally taken as $N^{2}=5 \times 10^{-4} \mathrm{~s}^{-1}$. Horizontal phase speeds relative to the mean flow is given by $c_{i}=\omega_{i} / k$. The phase propagation relative to the ground $\left(c_{g d}\right)$ between the times GWs displayed in Fig. 15 and 3 hours earlier can also be estimated from the 4D model output. Once the average speed of mean flow at wave 
activity regions is found, the phase speed relative to mean flow is obtained by subtracting the observed mean flow speed from the phase speed relative to the ground. The estimation of phase speeds from observation of 4D model dataset can thus be compared with the derived value of the phase speed $c_{i}=\omega_{i} / k$. Table 3 also summarizes derived phase speeds, mean wind speeds, observed phase speeds relative to ground and mean flow. Generally, the derived values of phases compares well with the observed phase speed relative to mean flow, indicating the waves follow a linear dispersion relationship at this level.

It is worth noting that vertical wavelengths of GWs shown in Figure 18 above the tropopause are much smaller than those below it. This is similar to a twofold decrease of vertical wavelengths of transient thermal heating-generated GWs during upward propagation (Salby and Garcia 1987). Decrease of vertical wavelengths of the GWs from troposphere to stratosphere can also be attributed to the variations of static stability across the tropopause in the model. In the following subsections, we will discuss in details the characteristics of the gravity waves in each experiment and their difference due to the difference in the background large-scale flow and baroclinicity.

\subsection{Sensitivity of gravity wave characteristics to the baroclinic growth rate}

\subsubsection{Sensitivity to the slope of the initial tropopause}

Experiment "BigS" ("LowS") employs a steeper (lower) initial tropopause that results in a stronger (weaker) jet streak and thus stronger (weaker) vertical wind shear (Fig. 2b-c). As expected, a faster (slower) growth rate of the baroclinic waves is simulated in BigS (LowS) than that in CNTL. 
The maximum wind speed at the initial time for BigS (LowS) is estimated to be 53 (39) $\mathrm{m} / \mathrm{s}$ which differs significantly from $47 \mathrm{~m} / \mathrm{s}$ in CNTL. While the upper-level jet streaks have been distorted, the difference between the wind speed maximum in BigS (LowS) and that in CNTL persists even after the surface cyclones have been well developed (Fig. 11; $102 \mathrm{~h}$ for CNTL, $72 \mathrm{~h}$ for BigS and $132 \mathrm{~h}$ for LowS). The flow, nearly straight initially, shows less curvature at these times at the trough region for LowS than for CNTL but roughly the same for BigS (as in CNTL). At the surface, the minimum SLP for CNTL, BigS and LowS reaches $948 \mathrm{hPa}, 948 \mathrm{hPa}$ and $953 \mathrm{hPa}$, respectively.

With the different initial geometry of the tropopause and subsequently different growth rate of the baroclinic waves, the characteristics of the mesoscale gravity waves generated from the upper-level jet streaks all differ significantly. Waves with a much shorter horizontal wavelength $\left(\lambda_{h} \sim 96.7 \mathrm{~km}\right)$ and a higher intrinsic frequency $(\omega=8.8 \mathrm{f}$, $\mathrm{f}=1.0 \times 10^{-4} \mathrm{~s}^{-1}$ ) are produced in the faster-growing baroclinic wave experiment BigS than those in CNTL ( $\left.\lambda_{h}=143.3 \mathrm{~km} ; \omega=3.70 \mathrm{f}\right)$. Conversely, gravity waves with slightly larger horizontal wavelength $(\sim 175 \mathrm{~km})$ and slightly lower intrinsic frequency (3.0f) are observed in LowS which has a smaller baroclinic growth rate than CNTL (Figs. 13-14, 18; Table 3).

\subsubsection{Sensitivity to tropospheric static stability}

Experiment "LowN" reduced the initial tropospheric PV and thus static stability in CNTL by half (Fig. 2d). Lower tropospheric PV before the 2-D PV inversion results in a much stronger jet streak $(56 \mathrm{~m} / \mathrm{s})$ and thus stronger vertical shear than CNTL at the initial time. Qualitatively consistent with the baroclinic index in Eq. (7), a stronger 
vertical shear and lower tropospheric satiability leads to the BWs growing 1.5 times faster than BWs in CNTL (Fig. 11a). The minimum SLP of the surface cyclone reaches $967 \mathrm{hPa}$ at $39 \mathrm{~h}$ in LowN while similar amplitude was reached around $102 \mathrm{~h}$ in CNTL. The BWs in LowN also have a slightly shorter zonal wavelength $(3600 \mathrm{~km})$ than CNTL $(\sim 3900 \mathrm{~km})$.

Consequently, a much shorter horizontal wavelength $(\sim 79.5 \mathrm{~km})$ and a much higher frequency (10.1f) of the GWs are simulated in LowN than in CNTL $(143.3 \mathrm{~km}$, 3.7f). Interestingly, this experiment which has the strongest baroclinic growth also produces the GWs with the highest intrinsic frequencies and the shortest horizontal wavelength among all experiments performed in this study.

\subsection{Gravity waves in the experiment with a lower tropospheric stability and a flatter}

\section{initial tropopause}

Experiment "LowNlowS" has a lower tropospheric stability and a flatter initial tropopause (Fig. 2g-h). The quasi-linear growth rate of BWs in LowNlowS ends around 90h, nine hours before CNTL case (Fig. 15b). Correspondingly, mature GWs are displayed earlier in LowNlowS (Fig. 15e, 111h). Growth rate curves of BWs in LowNlowS are slightly smaller than CNTL at quasi-linear growing stages (Fig. 14). Maximum wind speeds at jet streak core increase little from initial time (from $38.3 \mathrm{~m} / \mathrm{s}$ to $41 \mathrm{~m} / \mathrm{s}$ in LowNlowS) until 111h. Minimum SLP for LowNlowS reaches $951 \mathrm{hPa}$. LowNlowS achieve similar SLP and kinetic energy to CNTL at 102 hour when GWs have almost been initialized in the exit regions of jet streaks, which suggests that BWs in the two cases gain similar strength. 
GWs with nearly identical horizontal and vertical wavelengths and intrinsic frequency to those of the CNTL are produced in LowNlowS $\left(\lambda_{h}=143.2 \mathrm{~km} ; \omega=3.57 \mathrm{f}\right.$; Table 3). This sensitivity experiment suggests that mesoscale gravity waves generated in the exit regions of the upper-level jet-front system may have similar intrinsic frequency if BWs gain similar strength despite that the background BWs have slightly different growth rates. The strength of BWs is likely to have control over the GWs in addition to the effects of growth rates of BWs.

\subsection{Summary}

GWs with horizontal wavelength of 75-175 km generated near upper-level jetfronts are a universal phenomenon during the life cycles of BWs in our f-plane channel simulations. Generally, GWs occur at the time of upper level frontogeneis and jet streak distortion, which is also the later stage of quasi-linear growth and earlier stage of nonlinear growth for BWs. The synoptic flow patterns for these gravity waves is very consistent to that identified by UK87 from observational studies.

Sensitivity tests to the baroclinicity of the lager-scale baroclinic waves suggest that: the larger the growth rates of the baroclinic waves, the higher the intrinsic frequencies and the shorter the horizontal wavelengths of the mesoscale gravity waves. Increasing the initial slopes of the tropopause leads to stronger shear environment and subsequently faster growing baroclinic waves. The gravity waves generated during these baroclinic waves have shorter horizontal wavelengths and higher intrinsic frequency. Decrease of the slopes of the tropopause has opposite effects. Decreasing initial tropospheric static stability leads to stronger shear and lower tropospheric static stability. 
The subsequent baroclinic waves have a faster growth rate during the life cycles of which the gravity waves in the lower stratosphere have shorter horizontal wavelengths and faster intrinsic frequencies.

The experiment simultaneously changing tropospheric static stability and slopes of the tropopause could produce baroclinic waves with slightly small growth rate to CNTL. The subsequent jet-streak gravity waves have similar intrinsic frequency and wavelengths to those in CNTL which further suggests that the gravity wave characteristics are strongly controlled by the background baroclinic waves including the growth rate and strength of BWs.

We have also performed various experiments with different baroclinic growth rate through using a different Coriolis parameter (planetary rotation) before the PV inversion (not shown). Both the scales of the baroclinic waves and gravity waves in these experiments can vary significant with the change of planetary in a manner more complicated than those experiments described above. 


\section{FLOW IMBALANCE AND BALANCE ADJUSTMENT}

\subsection{Overview of flow imbalance diagnosis}

As discussed in Z04 which hypothesized balance adjustment to be responsible for generating the mesoscale gravity waves from the upper-tropospheric jet-front system, the flow imbalance ( $\triangle N B E$ ) produced during the baroclinic life cycles may act as a continuous forcing to upward propagating GWs. However, the residue of the nonlinear balance or $\triangle N B E$ comes not only from the localized region of imbalance but also from the highly unbalanced gravity waves resulting from the imbalance. The localized flow imbalance indicated by significantly large, positive $\triangle N B E$ at $7 \mathrm{~km}$ for all experiments is shown in horizontal plane (Fig. 20) and vertical cross sections (Fig. 21) at 9-12 hours before leading mature GWs seen in $13 \mathrm{~km}$ (Fig. 16). The timings are summarized in Table 2. It is worth noting that, with the uses of PV inversion in the initialization, the jetfront system is nearly perfectly balanced at the initial times. The evolution of the NBE residual from the initial balanced state to strong imbalance shown in Figs. 20-21 in all the sensitivity experiments is very similar to that of the CNTL demonstrated in Figs. 10-11 of Z04.

More specifically, Figure 21 demonstrates that imbalance maximizes immediately above the tropopause, i.e. $7 \mathrm{~km}$ for most cases. Regions of positive $\triangle N B E$ at $7 \mathrm{~km}$ collocate with a thermal ridge, which is caused by downward intrusion of warm air that originated from the stratosphere (Fig. 20). The upper level jet front systems represent the typical mesoscale environment of imbalance, which was discussed in section 3 . At the times when the imbalance is shown in Fig. 20, the baroclinic waves begin to enter the late stages of quasi-linear growth and early stages of nonlinear growth or saturation for most 

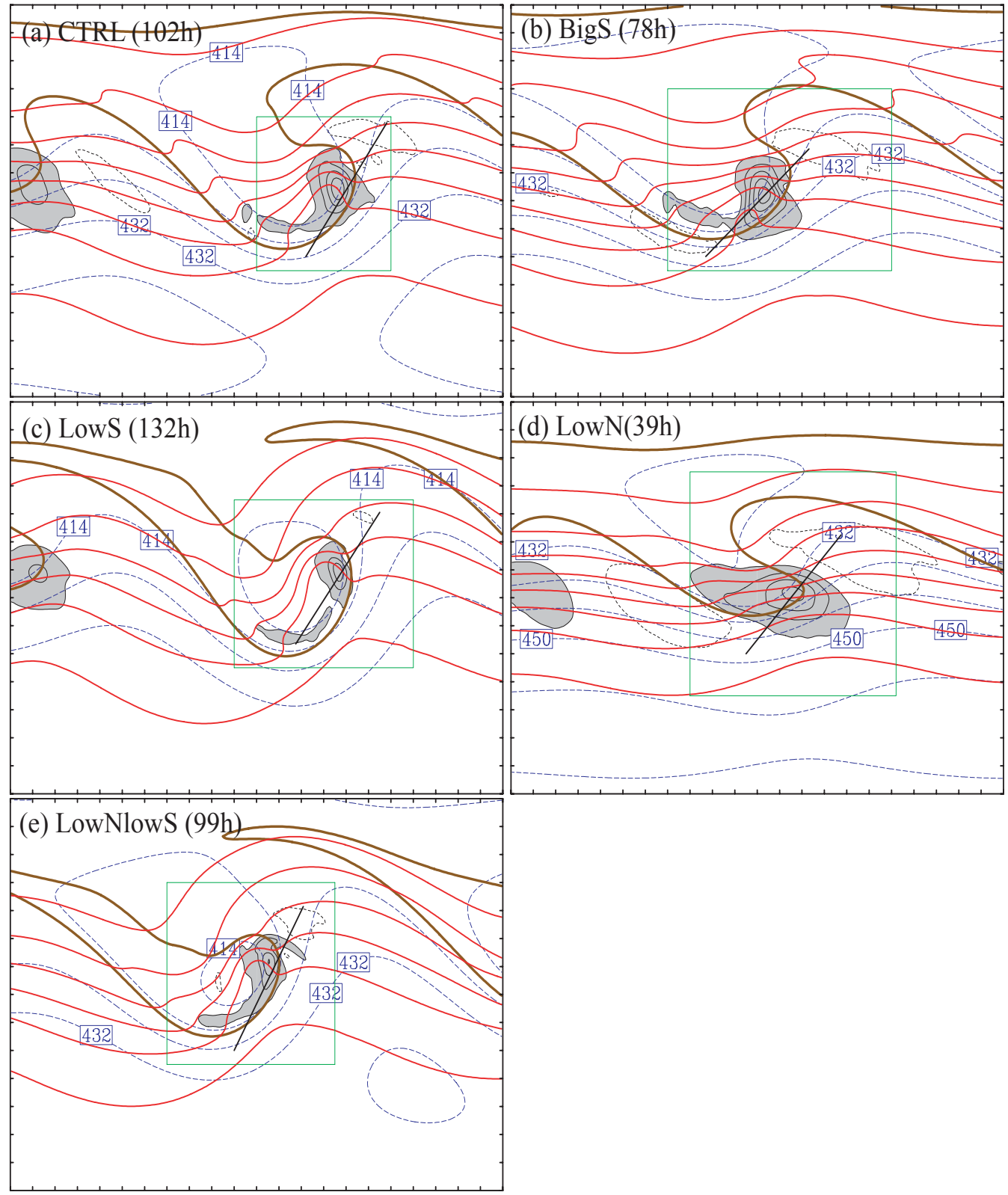

Figure 20: $\triangle N B E$ (shaded, solid, positive; dashed, negative. ci $=0.01 \times 10^{-8} \mathrm{~s}^{-2}$ ), pressure (blue solid lines, ci $=6 \mathrm{hPa}$ ) and $\theta$ (red lines, ci $=5 \mathrm{~K}$ ) at $7 \mathrm{~km} \mathrm{9-12} \mathrm{hours} \mathrm{before} \mathrm{GWs}$ are shown in Fig. 15. Timings are also summarized in Table 2. The distance between tick marks is $300 \mathrm{~km}$. 

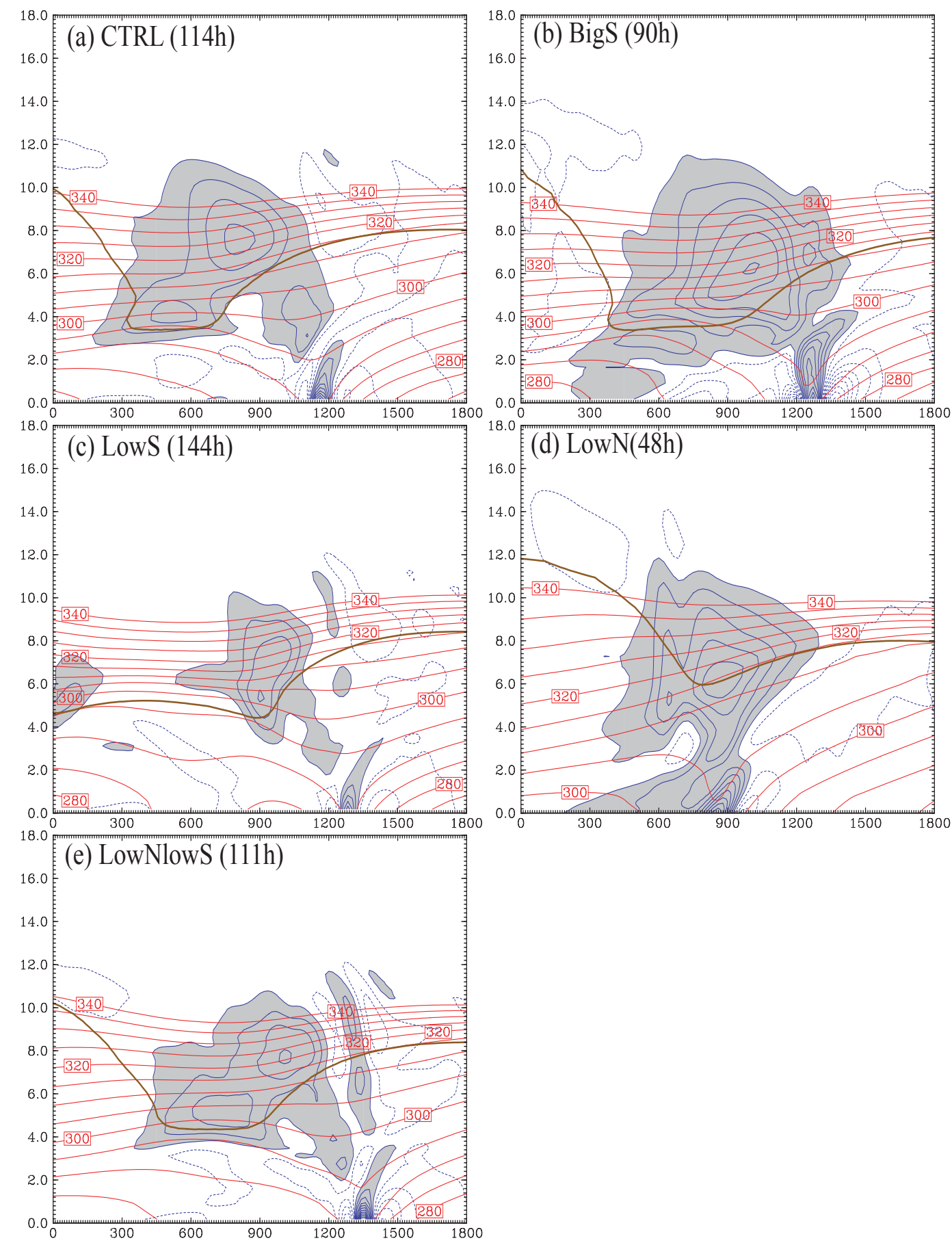

Figure 21: $\triangle N B E$ (shaded, solid, positive; dashed, negative. ci $=0.01 \times 10^{-8} \mathrm{~s}^{-2}$ ) and potential temperature (red lines, $\mathrm{ci}=5 \mathrm{~K}$ ) at the cross sections indicated by straight lines in Fig. 20. 
cases (refer to the big circles in Fig. 15). At this time, weak gravity wave signals become visible in $\triangle N B E$ field (Fig. 21 a,c and e) as well as divergence fields (Fig. 11), though it can hardly be seen in the vertical motion fields (not shown). The weak gravity wave signals appeared just downstream of the localized imbalance maxima near the tropopause (upper-level jet-front system) for all experiments. To track the intensity of the imbalance, we construct the time series of averaged imbalance at ten points with largest $\triangle N B E$ (Fig. 22). The time series of all cases are used to investigate the relationships between the GWs and the preceding imbalance.

Similar to evolution of minimum SLP perturbation (Fig. 13), the maximum residual of nonlinear balance equation increase continuously until late into the baroclinic wave life cycles after the quasi-linear growth phase and well into the nonlinear growth (saturation) phase. The increase of maximum localized imbalance is accompanied by scale contraction. $\triangle N B E$ at the times of mature gravity waves displayed in $13 \mathrm{~km}$ (Fig. 15) are also shown in Fig. 23. At these times, $\triangle N B E$ are filtered by subtracting smoothed $\triangle N B E$ from the original $\triangle N B E$ fields, which is the same technique that is used to obtain perturbation vertical velocity (Fig. 17-18). $\triangle N B E$ anomalies are plotted in Fig. 24 in the vertical cross sections indicated in Fig. 15. Wave signals are clearly demonstrated.

\subsection{Sensitivity of flow imbalance to baroclinic growth rate}

Gravity waves with higher (lower) frequencies appear near jet-front systems in BigS (LowS), which has steeper (flatter) slopes of the initial tropopause (see section 4.3.1). Growth rates of $\triangle N B E$ maximum are calculated from Fig. 22 and summarized in 


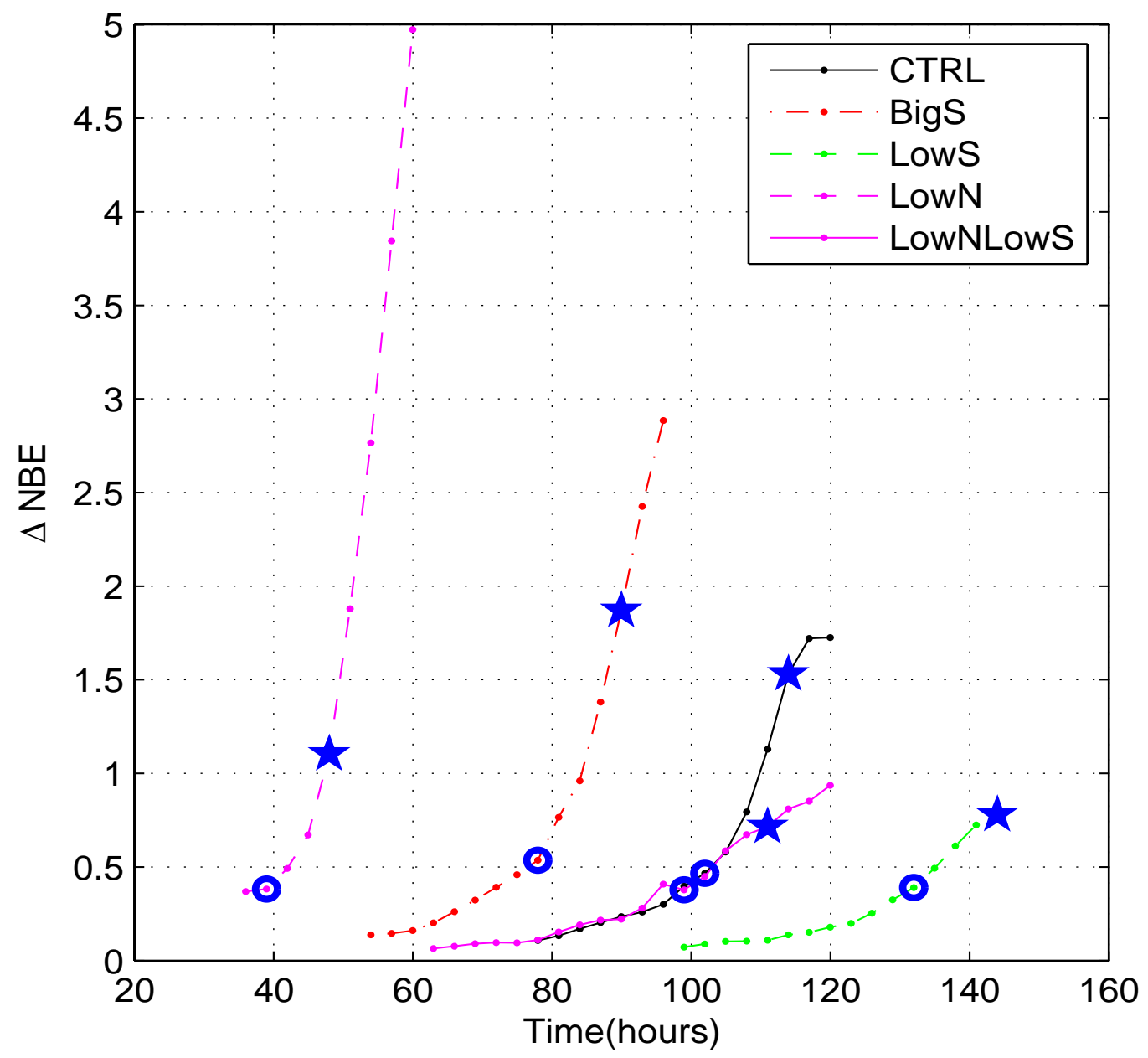

Figure 22: Time series of averaged $\triangle N B E\left(10^{-8} \mathrm{~s}^{-2}\right)$ at 10 points with largest values for 5 cases. 


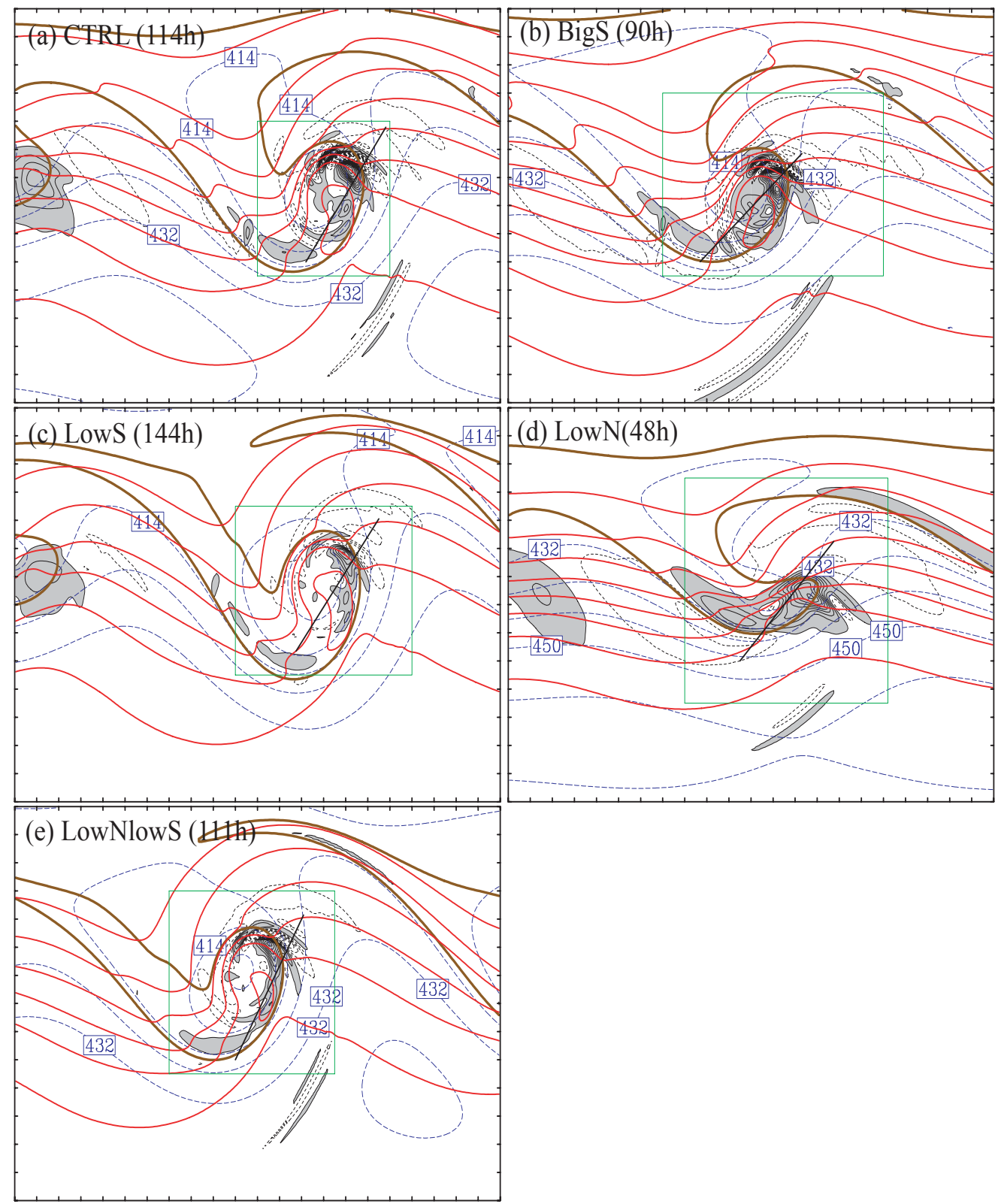

Figure 23: $\triangle N B E$ (shaded, solid, positive; dashed, negative. $\mathrm{ci}=0.01 \times 10^{-8} \mathrm{~s}^{-2}$ ) pressure (blue lines $\mathrm{ci}=6 \mathrm{hPa}$ ), and $\theta$ (red lines, $\mathrm{ci}=5 \mathrm{~K}$ ) at $7 \mathrm{~km}$ at the same times as GWs are shown in Fig. 15. Timings are also summarized in Table 2. The distance between tick marks is $300 \mathrm{~km}$. 

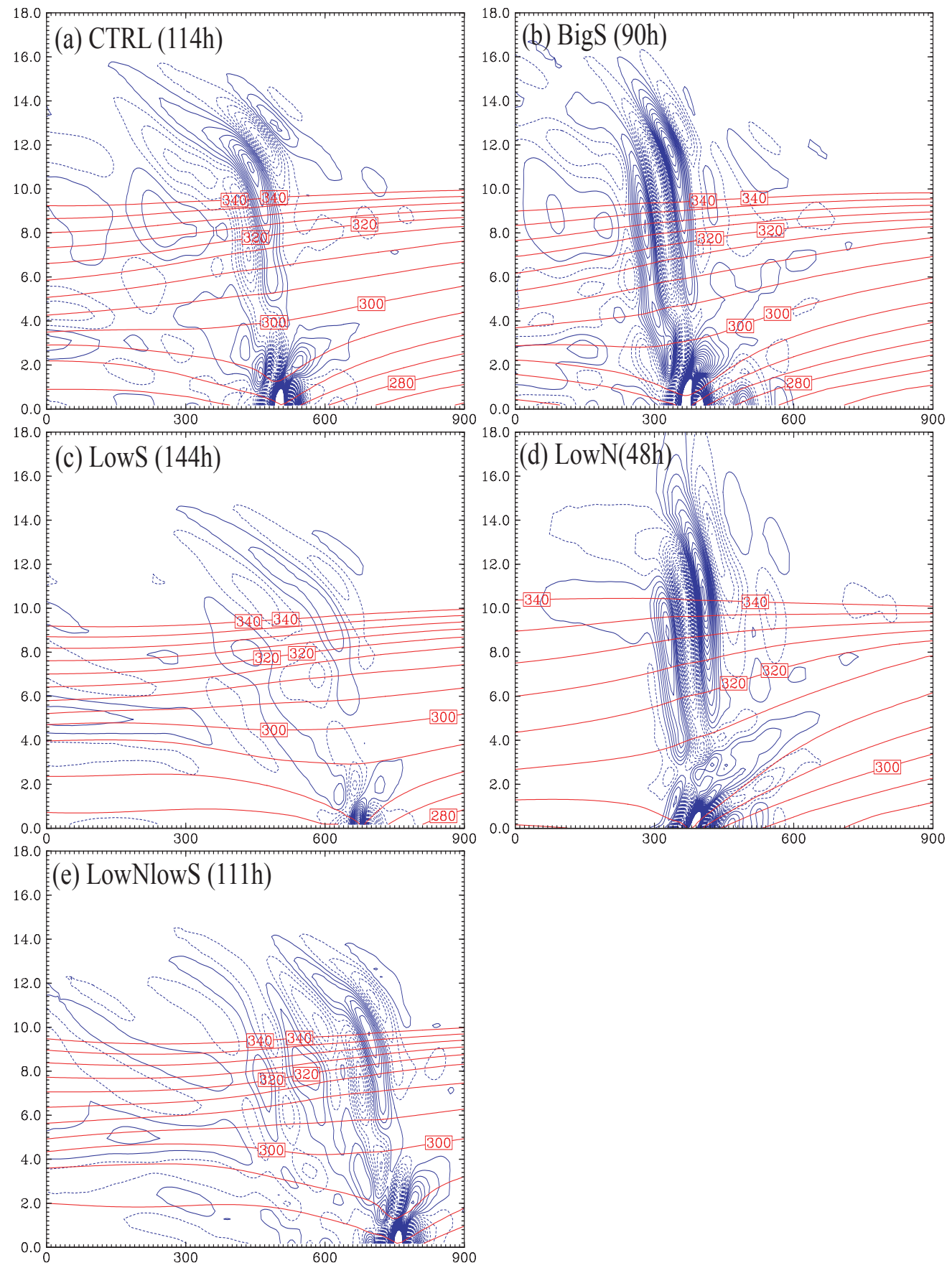

Figure 24: $\triangle N B E$ anomalies (blue lines, ci $=0.01 \times 10^{-8} \mathrm{~s}^{-2}$ ) and $\theta$ (red lines, ci $=5 \mathrm{~K}$ ). 
Table 3. Evolutions of $\triangle N B E$ maximum (Fig. 22) suggest that a faster (slower) developing BW also leads to a faster (slower) growing imbalance in BigS (LowS). Fastest growth of imbalance is seen in LowN, which has initial tropospheric static stability nearly half of that in CNTL. GWs with highest frequencies are produced in LowN (see section 4.3.2).

Tuning of tropopause geometry and static stability leads to the LowNlowS case with slightly smaller quasi-linear growth of the baroclinic waves than BWs in CNTL. Time evolutions of imbalance for them display similar growth rates (Fig. 22 and Table 3). Gravity waves with similar frequencies to CNTL are also produced in LowNlowS (section 4.4).

Since the growth of imbalance is directly related to the growth rate of the baroclinic waves, the gravity wave frequencies thus also correlate closely with the evolution and growth of preexisting flow imbalance, which is consistent with the analytical argument proposed by Plougonven and Zhang (2005, personal communications) discussed in section 3. However, the exact relationship between the growing imbalance and baroclinic growth rates can not be clearly identified for the current study. More specifically, GWs generated in jet-front systems with faster growing imbalance have higher intrinsic frequencies. The maximum imbalance curves and wave frequencies seems to suggest that wave frequencies are positively correlated to the growth of preexisting imbalance indicated by the residuals of the nonlinear balance equation. This correlation appears to justify using the scale separation argument (section 3) to derive the wave equation (eq. 11), which takes the imbalance as the forcing to the generation of gravity waves. 
Although faster growing baroclinic waves leads to shorter GW horizontal wavelengths, what selects the scales of gravity waves remains unclear. This failure can be partly attributed to the complications of background shear environments. For example, vertical wave length of gravity waves shown great variations when gravity waves propagate across the tropopause. In addition, strong imbalance also appears near surface fronts (Fig. 21 and 24). However, roles of surface fronts are hard to assess since they are integral parts of baroclinic waves. 


\section{SUMMARY AND DISCUSSION}

The generation and characteristics of mesoscale gravity waves near upper level jet-front systems during the life cycle of baroclinic waves are addressed numerically in this study. Five different life cycles of idealized baroclinic waves are simulated with a high-resolution mesoscale model (MM5) with 10-km grid spacing. The initial conditions for all the MM5 simulations consist of a small three-dimensional PV-disturbance at the tropopause of a two-dimensional baroclinic jet which is nonlinearly balanced through PV inversion of Davis and Emanuel (1991). The initial baroclinicity is modified as a result of using different tropospheric static stability and tropopause geometry for the initial twodimensional baroclinic jet. Consequently, the growth rate of the baroclinic waves, which is determined by the initial baroclinicity, differs significantly among these five experiments. After a short period of initial adjustment, the baroclinic waves in each experiment grow nearly linearly for a few days before the final nonlinear growth stage. The linear growth stage extends longer (up to 7 days) for slower growing baroclinic waves, but shorter (1-2 days) for faster growing cases.

Vertically propagating mesoscale gravity waves are generated universally in the exit regions of the upper-tropospheric jet streaks near the tropopause level at the later stage of quasi-linear growth and the early stage of nonlinear growth of baroclinic waves. The synoptic environments of gravity waves consist of surface fronts, upper level jetfronts and an upper level trough, consistent with previous studies of typical mesoscale gravity waves synthesized by UK87.

As demonstrated in Zhang (2004), the mesoscale gravity waves in the control simulation are closely related to the localized flow imbalance, which is defined as the 
residuals of the nonlinear balance equation. It is shown that this localized imbalance maximizes at a thermal ridge downstream of trough. Further diagnostics show that its surrounding mesoscale flow consists of unbalanced pressure, maximum gradient of divergence and vertical motions near the upper level jet front systems.

The low-stratospheric gravity waves generated from the upper-level jet streak in these experiments have a horizontal wavelength of 75-175 $\mathrm{km}$ and an intrinsic frequency of 3-9 times of the Coriolis parameter. Sensitivity tests to the growth rates of baroclinic waves reveal that: (i) Increasing the initial slopes of the tropopause leads to stronger jet streak and vertical shear which subsequently results in faster growing baroclinic waves. The gravity waves generated during the faster growing baroclinic waves have shorter horizontal wavelengths and lower intrinsic frequency. Decreasing of the slopes of the tropopause has opposite effects. (ii) Decreasing the initial tropospheric static stability leads to stronger shear. The subsequent baroclinic waves have faster growth rate. Gravity waves have shorter horizontal wavelengths and higher intrinsic frequencies. In general, the intrinsic frequency of these gravity waves appears to be positively correlated with the growth rate of the baroclinic waves. The faster the growth rate of the baroclinic waves, the higher the intrinsic frequency of the gravity waves.

Slightly smaller growth rates of the baroclinic waves are found in the experiment with similar initial baroclinicity but with significantly different initial tropospheric static stability and tropopause geometry. The simulated mesoscale gravity waves in this case have similar frequencies. It is suggested that the strength of baroclinic waves is likely to have control over the gravity waves in addition to the effects of growth rates of BWs. 
Diagnosis of flow imbalance provide insight into generation of gravity waves. Time series of maximum $\triangle N B E$ show that imbalance continuously increases while the gravity waves have been generated. Diagnosis of growing imbalance in the five cases suggest that the growth rate of the imbalance is directly related to the growth rate of baroclinic waves and thus the frequency of primary gravity waves of interests. In case of faster growing imbalance, gravity waves have higher frequencies. Experiments with similar growth rate of baroclinic waves suggest that imbalance is also positively correlated to frequencies of gravity waves.

This study presents merely a preliminary analysis on the relation between mesoscale gravity waves and the large scale environment and flow imbalance. It is also emphasized that the analysis are valid for the idealized initial conditions and $\mathrm{f}$ plane models. Although frequencies and wavelengths of gravity waves are found to be closely related to the growth rate of the background baroclinic waves, it remains unclear what else may determines the temporal and spatial scales of the gravity waves. Selection of the gravity wave scales and their relation to background mesoscale flow shall be an emphasis of the future work on this subject. 


\section{REFERENCES}

Allen, J. S., 1991: Balance equations based on momentum equations with global invariants of potential enstrophy and energy. Journal of Physical Oceanography, 21, $265-276$

Badger, J. and B. J. Hoskins, 2001: Simple initial value problems and mechanisms for baroclinic growth. Journal of the Atmospheric Sciences, 58, 38-49.

Bannon, P., 1995: Hydrostatic adjustment: Lamb's problem. Journal of the Atmospheric Sciences, 52, 1743-1752.

Blumen,W., 1972: Geostrophic adjustment. Reviews of Geophysics and Space Physics, 10, 485-528.

Bosart, L., W. Bracken, and A. Seimon, 1998: A study of cyclone mesoscale structure with emphasis on a large-amplitude inertia-gravity waves. Monthly Weather Review, 126, $1497-1527$.

Cahn, A., 1945: An investigation of the free oscillations of a simple current system. Monthly Weather Review, 119, 1929-1952.

Charney, J., 1952: The use of primitive equations of motion in numerical prediction. Tellus, 7, 22-26.

Davis, C. and K. Emanuel, 1991: Potential vorticity diagnosis of cyclogenesis. Monthly Weather Review, 119, 1929-1952.

Farrell, B. F., 1982: The initial growth of disturbances in baroclinic flows. Journal of the Atmospheric Sciences, 39, 1663-1686.

Fritts, D. C. and M. J. Alexander, 2003: Gravity wave dynamics and effects in the middle atmosphere. Reviews of Geophysics, 41, 1003-1063.

Gill, A., 1982: Atmosphere-Ocean Dynamics. Academic Press, 622 pp. 
Griffiths, M. and M. Reeder, 1996: Stratospheric inertia-gravity waves generated in a numerical model of frontogenesis. I: Model solutions. Quarterly Journal of the Royal Meteorological Society, 122, 1153-1174.

Guest, F. M., R. M. Reeder, C. J. Marks, and D. J. Karoly, 2000: Inertia gravity waves observed in the lower stratosphere over Macquarie Island. Journal of the Atmospheric Sciences, 57, 737-752.

Hakim, G., 2000: Role of nonmodal growth and nonlinearity in cyclogenesis initial-value problem. Journal of the Atmospheric Sciences, 57, 2951-2967.

Haltiner, G. J. and R. T. Williams, 1980: Numerical Prediction and Dynamic Meteorology, John Wiley \& Sons, Inc, $2^{\text {nd }}$ Edition, 477pp.

Kaplan, M., S. Kock, Y.-L. Lin, R. Weglarz, and R. Rozumalski, 1997: Numerical simulations of a gravity wave event over CCOPE. Part I: The role of geostrophic adjustment in mesoscale jetlet formation. Monthly Weather Review, 125, 1181-1211.

Keyser, D. and M. Shapiro, 1986: A review of the structure and dynamics of upper-level frontal zones. Monthly Weather Review, 114, 452-499.

Koch, S. and P. Dorian, 1988: A mesoscale gravity wave event observed over CCOPE. Part 3: Wave environment and probable source mechanisms. Monthly Weather Review, 116, 2570-2562.

Koch, S. and C. O'Handley, 1997: Operational forecasting and detection of mesoscale gravity waves. Weather and Forecasting, 12, 253-281.

Koch, S., F. Zhang, M. Kaplan, and Y. Lin, 2001: Numerical simulation of a gravity wave event observed during CCOPE. Part 3: Mountain-plain solenoids in the generation of the second wave episode. Monthly Weather Review, 129, 909-932.

Lindzen, R. and B. Farrell, 1980: A simple approximate result for the maximum growth rate of baroclinic instabilities. Journal of the Atmospheric Sciences, 37, 1648-1654. 
O’Sullivan, D. and T. Dunkerton, 1995: Generation of inertia-gravity waves in a simulated life cycle of baroclinic instability. Journal of the Atmospheric Sciences, 52, $3695-3716$.

Pierrehumbert, R. and K. Swanson, 1995: Baroclinic instability. Annual Review of Fluid Mechanics, 27, 419-467.

Powers, J. and R. Reed, 1993: Numerical model simulations of the large-amplitude mesoscale gravity-wave event of 15 December 1987 in the central Unite States. Monthly Weather Review, 121, 2285-2308.

Raymond, D., 1992: Nonlinear balance and potential-vorticity thinking at large Rossby number. Quarterly Journal of the Royal Meteorological Society, 118, 987-1015.

Reeder, M. and M. Griffiths, 1996: Stratospheric inertia-gravity waves generated in a numerical model of frontogenesis. II: Wave sources, generation mechanism and momentum flux. Quarterly Journal of the Royal Meteorological Society, 122, 11751195.

Rossby, C., 1937: On the mutual adjustment of pressure and velocity distributions in certain simple current systems I. Journal of Marine Research, 1, 15-28.

Rotunno, R. and J.-W. Bao, 1996: A case study of cyclogensis using a model hierarchy. Journal of the Atmospheric Sciences, 124, 1051-1066.

Rotunno, R., W. Skamarock, and C. Snyder, 1994: An analysis of frontogenesis in numerical simulations of baroclinic waves. Journal of the Atmospheric Sciences, 51, $3373-3398$.

Salby, M. L. and R. R. Garcia, 1987: Transient response to localized episodic heating in the tropics, part i, excitation and short-time near-field behavior. Journal of the Atmospheric Sciences, 44, 458-498.

Simmons, A. and B. Hoskins, 1978: The life cycles of some nonlinear baroclinic waves. Journal of the Atmospheric Sciences, 35, 414-432. 
Stone, P., 1978: Baroclinic adjustment. Journal of the Atmospheric Sciences, 35, 561571.

Uccellini, L. and S. Koch, 1987: The synoptic setting and possible source mechanisms for mesoscale gravity wave events. Monthly Weather Review, 115, 721-729.

Wandishin, M., J. W. Nielsen-Gammon, and D. Keyser, 2000: A potential vorticity diagnostic approach to upper-level frontogenesis within a developing baroclinic wave. Journal of the Atmospheric Sciences, 57, 3918-3938.

Zhang, F., 2004: Generation of mesoscale gravity waves in the upper-tropospheric jet front systems. Journal of the Atmospheric Sciences, 61, 440-457.

Zhang, F. and S. E. Koch, 2001: Numerical simulation of a gravity wave event observed during CCOPE. Part 2: Wave generation by an orographic density current. Monthly Weather Review, 128, 2777-2796.

Zhang, F., S. E. Koch, and M. L. Kaplan, 2000: A survey of unbalanced flow diagnostics and their application. Advances in Atmospheric Sciences, 17, 165-173.

Zhang, F., S. E. Koch, C. A. Davis, and M. L. Kaplan, 2001: Wavelet analysis and the governing dynamics of a large-amplitude gravity wave event along the east coast of the United States. Quarterly Journal of Royal Meteorological Society, 127, 2209-2245. 


\section{VITA}

Shuguang Wang was born in Zhongyang, Anhui Province, China on June, 1978. He graduated from the Nanjing University in June 2000 with a Bachelor of Science degree in atmospheric sciences. In August 2003, he began graduate school at Texas A\&M University in the Department of Atmospheric Sciences and received a Master of Science degree in atmospheric sciences in December 2005. Shuguang Wang can be contacted at his permanent address at 101 Front Street Apt 109, College Station, Texas, 77840. 